\title{
On the nature, formation and diversity of particulate coherent structures in Microgravity Conditions and their relevance to materials science and problems of Astrophysical interest
}

\author{
Marcello Lappa ${ }^{1}$ \\ ${ }^{1}$ Department of Mechanical and Aerospace Engineering, University of Strathclyde, James Weir \\ Building, 75 Montrose Street, Glasgow, G1 1XJ, UK - email: marcello.lappa@strath.ac.uk, \\ marlappa@unina.it
}

\begin{abstract}
Different phenomena related to the spontaneous accumulation of solid particles dispersed in a fluid medium in microgravity conditions are discussed, with an emphasis on recent discoveries and potential links with the general field of astrophysical fluid-dynamics on the one hand, and with terrestrial applications in the field of materials science on the other hand. With special attention to the typical physical forces at play in such an environment, namely, surface-tension gradients, oscillatory residual gravity components, inertial disturbances and forces of an electrostatic nature, specific experimental and numerical examples are presented to provide inputs for an increased understanding of the underlying cause-and-effect relationships. Studying these systems can be seen as a matter of understanding how macroscopic scenarios arise from the cooperative behaviour of sub-parts or competing mechanisms (nonlinearities and interdependencies on various spatial and temporal scales). Through a critical assessment of the properties displayed by the resulting structures (which appear in the form of one-dimensional circuits formed by aligned particles, planar accumulation surfaces, three-dimensional compact structures resembling "quadrics", microcrystallites or fractal aggregates), we discuss a possible classification of the related particle attractors in the space of parameters according to the prevailing effect.
\end{abstract}

Keywords: Particle dynamics and self-assembly, fluids, thermocapillary, thermogravitational and thermovibrational convection, hydrodynamic and electrostatic forces.

\section{Introduction}

A microgravity environment provides a unique laboratory in which scientists can observe and explore physical events, phenomena, and processes that are normally masked by the effects of Earth's gravity. For example, crystal growth from melt and solutions, capillary effects, multiphase flow, diffusive-transport processes, and suspensions can under normal gravity conditions be dominated by effects such as buoyancy, convection and sedimentation.

Because gravity's effect on fluids is so strong, scientists cannot determine what effect other (still poorly known) forces are having on fluid behaviour. Many of these forces become dominant in microgravity, allowing scientists to observe them without the competing influence of gravity. This 
means that from a practical point of view, research conducted in microgravity is increasing our understanding of physics to provide a foundation for predicting, controlling and improving a vast range of technological processes (Lappa, 2004).

Among the subjects currently under investigation in this environment (especially on the International Space Station, ISS, where a variety of devices and laboratories are available for the analysis of a range of subjects in fluid-dynamics, chemical engineering, materials science, biotechnology and bioengineering) of special interest are the dynamics of complex fluids. Such fluids are binary mixtures that have a coexistence between two phases, a fluid (in general, it is a liquid, although it might also be a gas in microgravity conditions) and a solid (in the form of dispersed fine particles).

These special fluids are known to exhibit unusual responses to applied force fields due to the geometrical constraints that the phase coexistence imposes and/or other mechanisms of various natures. The resulting macroscopic properties depend on several fundamental factors which play a role at the microscopic (particle-size) scale: collisions, particle interactions, viscous effects (drag), and inertia. At the same time, the macroscopic appearance can be also linked to more complex issues such as bulk convection, related chaotic dynamics, clustering on multiple length scales, and the interplay between these opposite tendencies.

The breath of practical applicability of such studies is large.

In many industrial processes involving particle formation or solids handling, particles have a tendency to stick together or to accumulate in specific regions of the physical space. In protein crystallization processes, macromolecules attach one another due to the supersaturated environment they are in (Carotenuto et al., 2002; Lappa, 2011). Suspension polymerization processes go through a "sticky-phase" with significant agglomeration levels (Yuan et al., 1991). In colloidal systems a variety of interactions can cause preferential concentration, and stabilization of colloids is a central issue (Klimpel and Hogg, 1986; Ozaki et al., 1988; Ivanov and Kralchevsky, 1997).

The main motivation underpinning such interest can be linked directly to the resulting spatial structure of the blend components. This structure essentially determines the mechanical properties and rheology of such mixtures (Utracki, 1983; Lee and Park, 1994; Mason, 1999; Roths et al., 2002). Similar concepts can be applied to immiscible metal alloys; the two-phase liquid consists of dispersed particles (the socalled minority phase) in a matrix liquid and the quality of the dispersion alloys is defined by the degree of homogeneity of the minority-phase distribution. The strength of these alloys and many other composite materials can be improved if a uniform dispersion of fine particles in the matrix is achieved (Ratke, 1992, Prinz and Romero, 1993; Lappa, 2005; Deng and Chawla, 2006).

Beyond typical industrial processes, agglomeration plays a role in diverse bio-related applications such as blood flow dynamics and bio-molecular cross-linking of particles (Han et al., 2003; Hiddessen et al., 2004).

As illustrated by several authors (Lappa et al., 2004; Saeedi et al., 2006; Huang et al., 2004; Balvin et al., 2009; Herrman et al., 2009), last but not least, all these phenomena and associated studies are 
also relevant to recent nanotechnologies and related products. Self-assembly and induced particle clustering are emerging as one of the main methods for construction of heterogeneous systems consisting of multiple component types in nano- and micro-scales. The self-assembly concepts (and resulting technologies used for system integration) involves preparation of parts that can recognize and bind to each other or form a template, and perfection of procedures that allow for high yield self-assembly of these parts into a system.

Obviously, for an agglomeration or clustering event to occur, matter (primary particles and/or agglomerates) need to be attracted to specific regions of the considered fluid domain first.

As shortly discussed in the following, such motion or "attraction" may be induced by different causes.

In the Earth's gravitational field the usual differences in density cause rapid spatial separation of components with different densities through sedimentation or flotation (as an example, in the typical casting processes discussed before, this prevents a homogeneous distribution of particles in the matrix, thereby limiting the industrial exploitation of many alloys, Prinz and Romero, 1993).

As shown by microgravity-based experimentation, however, even in the absence of gravity, other forces can cause particle demixing and/or agglomeration (Ratke et al., 1996). These forces include: surface-tension gradients at liquid-liquid or liquid-gas interfaces, g-jitters (vibrations with various frequency and amplitude), shear stresses present in the fluid hosting the particles or other forces produced by Brownian or electrostatic effects.

Also particle inertia can be a possible cause. The dynamics of inertial (non-Brownian) particles are, in general, dissipative even if the background flow is laminar and regular (Bec, 2005). Moreover, it is known that due to inertia, the transport of particles in incompressible flows shows properties typical of a compressible fluid (Balkowsky et al., 2001; Benczik et al., 2002; Bec et al., 2007). It has been argued that these general properties can produce significant fluctuations of concentration and can, hence, support mechanisms for particle segregation and ordering (out of an initially homogeneous distribution) even if surface or body forces are negligible or absent.

Remarkably, the growing interest for these aspects is not limited to the realm of technology and related industrial applications. Relevant theoretical links can be also identified with a variety of problems of atmospheric, geophysical and astrophysical interest, such as rain generation (Babiano et al., 2000; Devenish et al., 2012), the advection of volcanic ash in the atmosphere (Haszpra and Tél, 2011), the formation of three-dimensional particulate coherent structures in hurricanes (Sapsis and Haller, 2009) and the mechanisms at play in the early stages of formation of asteroids and planets (Bracco et al., 1999).

Among them, the last subject is of a special interest because for decades theorists have had problems in elaborating models to explain the growth of asteroids and planets starting from a population of small dust grains in a protoplanetary gas-and-dust disk.

A crucial step in planet formation is the growth of solid bodies in the sub-millimeter to meter size range: too large to condense directly from the gas phase and too small to interact meaningfully through mutual gravitation (which would allow investigators to study this process entirely in the 
framework of the well-known N-body problem, see, e.g., Kokubo and Ida, 2000). The existence of planets in our solar system demands that some growth process once operated in that size regime, but the underlying cause-and-effect relationships have not been clarified yet (the major problem is to reconcile the time scales for growth by accumulation of objects of the size of gas-giant planets and the estimated lifetime of the Solar System; these time scales depend quite crucially on both the conditions prevailing in the ambient solar nebula and the details of the agglomeration process).

Motivated by these arguments, in the present paper we survey some still poorly known mechanisms recently identified as possible sources of particle segregation and self-assembly phenomena in microgravity conditions. Obviously, the focus is on the typical physical forces at play in such an environment and in the orbiting laboratories currently available for these studies such as the ISS (namely, inertia, oscillatory residual gravity components, vibration-driven interactions and forces of an electrostatic nature). The discussion progresses with the aid of specific experimental and numerical examples (some taken from the recent literature, others expressly conceived to support this work) presented here to provide inputs for an increased understanding of the underlying mechanisms.

As the reader might have realized at this stage, the main motivation at the root of the present survey is the express intent of using the main results provided by recent microgravity research to promote contacts and interdisciplinary knowledge exchange between the communities of materials engineers on the one hand, and geophysical and astrophysical fluid physicists on the other hand.

\section{Relevant Physical Effects and Quantities}

\subsection{Characteristic Time scales and Non-dimensional Numbers}

The relatively synthetic review of the literature provided in the introduction indicates that the general pattern-formation problem in solid-liquid systems is rich, and several issues are embedded in it.

Attempting to assemble a simple, physically intuitive and reasonably self-contained discussion of such phenomena in microgravity is not an easy task. Here, we concentrate on two main categories of solid particle attractors which have enjoyed a growing interest over recent years, one coming under the general heading of "inertial phenomena" (requiring solid/fluid density difference, finiteness of particle sizes and masses and some mean background flow transporting otherwise still particles, particle interactions being not necessary) and the other intimately related to the existence of inter-particle forces (requiring neither solid/liquid density difference nor the existence of a "background flow").

Further characterization of these phenomena requires the introduction of some necessary concepts and notions. Such a theoretical melange is necessary for a better recognition, definition and characterization of the aforementioned phenomena. Also, these general considerations facilitate the 
subsequent introduction of more complex notions and will significantly help the reader in the understanding of the theoretical explanations and arguments given throughout this survey.

To do so, in particular, we start from the cardinal concept of "characteristic time".

For all phenomena of convective nature leading to the emergence of a background flow able to produce macroscopic transport of a dispersed phase, a characteristic convective time must be introduced. For classical (thermal) buoyancy convection, for instance, most conveniently, this time can be defined as:

$$
\tau_{g}=\frac{v}{g \beta_{T} \Delta T(L)}
$$

where $g$ is the gravity acceleration, $\beta_{\mathrm{T}}$ is the fluid thermal expansion coefficient, $v$ is the kinematic viscosity, $\Delta \mathrm{T}$ a characteristic temperature difference established across the considered system and $\mathrm{L}$ a representative length.

In microgravity conditions, where steady gravity is absent, this reference temporal scale must be replaced by alternate (equivalent) forms depending on the specific case considered.

As an example, if thermal convection is being produced by "vibrations" in place of steady gravity, the characteristic time shall take into account in some way the "properties" of the considered vibrations.

Without loss of generality, here we assume shaking of the considered geometrical system along a given direction ( $\underline{\hat{n}}$ is the related unit vector) in the form $\underline{s}^{l a b}(t)=b \sin (\omega \mathrm{t}) \underline{\hat{n}}$ where $s^{\text {lab }}$ is the system time-varying displacement as seen by an observer in the fixed (laboratory) frame, $b$ is its amplitude and $\omega=2 \pi f$ is the related angular frequency. Taking into account that its instantaneous acceleration will read accordingly $\underline{g}(t)=g_{\omega} \sin (\omega \mathrm{t}) \underline{\hat{n}}$ where $g_{\omega}=\mathrm{b} \omega^{2}$, a straightforward extension of the characteristic convective time (eq. (1)) to the case of thermovibrational convection can be obtained by simply replacing g with $g_{\omega}=\mathrm{b} \omega^{2}$, i.e.

$$
\tau_{t v c}=\frac{v}{\mathrm{~b} \omega^{2} \beta_{T} \Delta T(L)}
$$

(because vibrating a system with frequency $f$ and displacement amplitude $b$ corresponds to a sinusoidal gravity modulation with the same frequency and acceleration amplitude $b \omega^{2}$ and vice versa, hereafter we will use the terms "forcing", "gravity modulation", "periodic acceleration", "system vibration" and g-jitter as synonyms).

The following remark should be noted in interpreting and using the above temporal scales (eqs. (1) and (2)). First, they have been cast in terms of acceleration amplitude; second they have been based on the idea that the fluid undergoes a density change under the influence of a temperature gradient. If the considered flow is produced by surface-tension effects, it is obvious that the dependence of the characteristic time on a body acceleration as seen in eqs. (1) and (2) shall be replaced by an 
equivalent dependence on the considered surface forces. If such forces are still produced by a temperature gradient (which leads us to the well-known case of thermocapillary or "Marangoni" convection), then, the characteristic convective time can be given the expression

$$
\tau_{\sigma}=\frac{\mu L}{\sigma_{T} \Delta T}
$$

where $\mu$ is the fluid dynamic viscosity and $\sigma_{\mathrm{T}}$ the derivative of the surface tension with respect to temperature.

As transport of heat and momentum by diffusion also plays a role in the development of a largescale flow (be it of buoyancy or surface-tension nature), relevant characteristic times (denoted by $\tau_{\alpha}$ and $\tau_{v}$, respectively) must be also introduced to account for such phenomena:

$$
\begin{gathered}
\tau_{\alpha}=\frac{L^{2}}{\alpha} \\
\tau_{v}=\frac{L^{2}}{v}
\end{gathered}
$$

where $\alpha$ is the fluid thermal diffusivity. Furthermore, if dispersed electric charges are present in the considered liquid, a characteristic time must be defined for the microscopic transport of such charges as well:

$$
\tau_{q}=\frac{\varepsilon \varepsilon_{0}}{\gamma}
$$

In practice, $\tau_{\mathrm{q}}$ can be regarded as a measure of the time required for the dispersed electrostatic charge to be dissipated by conduction to some electrical ground or sink region (this parameter is generally referred to as the "charge relaxation time", where $\varepsilon_{o}$ the permittivity of vacuum and $\varepsilon$ and $\gamma$ are the nondimensional dielectric constant and the electric conductivity of the considered fluid, respectively).

Obviously, the dispersed (solid) phase even introduces additional degrees of freedom in the considered problem (particle shape, size and density). Associated dimensional quantities are the particle density $\rho_{\mathrm{P}}=\left(\xi \rho_{\mathrm{F}}\right)$ (where $\xi$ is the ratio of the solid matter to the fluid density), and the particle radius $\mathrm{R}$ (for simplicity, here we assume particles to be perfectly spherical). The related so-called particle "relaxation time" $\left(\tau_{\text {part }}\right)$ is generally defined in the literature as:

$$
\tau_{\text {part }}=\frac{2}{9} \frac{R^{2}}{v}
$$


Moreover, to properly account for the effect of vibrations on particle motion, another relevant time scale must be considered, namely, the characteristic vibration time:

$$
\tau_{\omega}=\frac{2 \pi}{\omega}
$$

What makes really worth the effort of introducing so many quantities is the possibility to get additional insights into the phenomena of interest by considering the nondimensional numbers which emerge taking the ratios of such temporal scales. Indeed, a variety of possible combinations are possible, which can be used to "measure" the relative importance of different effects.

As an example the ratios:

$$
\begin{aligned}
& \frac{\tau_{\alpha}}{\tau_{g}}=R a=\frac{g \beta_{T} \Delta T(L)^{3}}{v \alpha} \\
& \frac{\tau_{\alpha}}{\tau_{t v c}}=R a_{\omega}=\frac{\mathrm{b} \omega^{2} \beta_{T} \Delta T(L)^{3}}{v \alpha} \\
& \frac{\tau_{\alpha}}{\tau_{\sigma}}=M a=\frac{\sigma_{T} \Delta T L}{\mu \alpha}
\end{aligned}
$$

lead to the well-known classical Rayleigh, vibrational Rayleigh and Marangoni numbers, respectively, traditionally used to measure the "strength" of the related flows (of thermogravitational, thermovibrational or thermocapillary nature, respectively). The ratio of the two characteristic times for heat and momentum transport at microscopic level:

$\frac{\tau_{\alpha}}{\tau_{v}}=\frac{v}{\alpha}=\operatorname{Pr}$

in turn, gives rise to the well-known Prandtl number, which may be regarded as a specific "signature" of the considered fluid (as it depends on thermodynamic properties only). A less known, but still relevant nondimensional number is given by the ratio of the charge relaxation time (eq. 6) to the viscous diffusion time (eq. 5):

$$
\frac{\tau_{q}}{\tau_{v}}=Q=\frac{\varepsilon \varepsilon_{0}}{\gamma} \frac{v}{L^{2}}
$$

It can be considered as a "measure" of the ability of the considered flow to "retain" electric charges eventually produced in the fluid by large-scale flow and related "frictional" effects (we will come back to this important concept later). 
While the above nondimensional parameters are used in general to characterize the macroscopic or "background" flow developing in the fluid hosting the dispersed particles, the nondimensional numbers obtained as the ratio of $\tau_{\text {part }}$ (eq. (7)) to some other relevant temporal scales, give useful information into the motion of the particles per se. Relevant characteristic parameters along these lines are (see, e.g., Lappa, 2013a):

$$
\begin{aligned}
& S t=\frac{\tau_{\text {part }}}{\tau_{v}}=\frac{2}{9} \frac{R^{2}}{L^{2}}=\frac{\eta}{\xi-1} \operatorname{Pr} \\
& S t_{v}=\frac{\tau_{\text {part }}}{\tau_{\omega}}=\frac{1}{9 \pi} \frac{R^{2} \omega}{v}=\frac{1}{2 \pi} \frac{\eta \varpi}{\xi-1} \quad, \text { where } \varpi=\frac{\omega L^{2}}{\alpha}
\end{aligned}
$$

usually referred to as the particle Stokes number and vibrational Stokes number, respectively, where

$$
\eta=\frac{\tau_{\text {part }} \alpha}{L^{2}}(\xi-1)
$$

is a general associated non-dimensional quantity often used in the literature related to flows of thermal origin (see, e.g., Pushkin et al., 2011) to account for the combined effect of particle inertia and drag.

The above particle-related nondimensional numbers have a precise physical significance as well. As outlined in the introduction, it has been well known in the fluid mechanical literature that small but finite sized particles with densities different from that of the surrounding fluid may deviate from the fluid trajectories and that these deviations, accrued over time, can lead to nonconservative trajectories in otherwise volume-preserving flows.

In such a context, while, the limit $\mathrm{St} \rightarrow 0$ would represent the condition for which inertial particles recover the motion of fluid particles and no clusterization is expected (in other words, particles simply moving with the local fluid velocity as mere ideal Lagrangian tracers), the opposite limit St $>>1$, should be regarded as a situation in which a particle would become less and less influenced by the velocity field. Between such limit conditions, a range of intermediate values of $\mathrm{St}(<1)$ exists where the above-mentioned nonconservative (dissipative) effects can produce particle demixing and/or accumulation phenomena.

The other nondimensional number, $\mathrm{St}_{\mathrm{v}}$, the particle vibrational Stokes number (eq. 15), is generally used to account for the hydrodynamic (attractive or repulsive) interactions promoted by the application of vibrations to a concentrated suspension of particles (as we shall outline in more detail in Sect. 5).

For the sake of clarity, in the following, first we restrict our attention to the case of dilute suspensions where particle interference is expected to play no role (the main source of selfassembly phenomena being inertial dissipative effects, with particles being transported by thermal convection produced by forces of various natures). Later, we consider specific examples where such phenomena must be expressly ascribed to inter-particle forces. 


\subsection{The Notion of "Vorticity" and its role in Particle Dynamics}

Throughout the manuscript, useful additional insights into the considered dynamics are sought via some microphysical reasoning based on the introduction of the concept of flow "vorticity", mathematically defined as the curl of the carrier fluid velocity field:

$\underline{\zeta}=\underline{\nabla} \wedge \underline{V}$

Such quantity is expressly invoked and used to elaborate a specific mathematical formalism and some associated physical arguments.

Perhaps, it is worth recalling at this stage that a scenario in which vorticity at small or large scales can mediate particle accumulation phenomena is not a new concept in the literature.

The role of vorticity in promoting particle segregation for laminar flows or in conjunction with turbulence has been already recognized by several investigators. As an example, the tendency of heavy inertial particles to leave the neighbourhood of a vortex was identified as the main mechanism by which vortical structures cause concentration nonuniformities in typical technological processes since the works by Eaton and Fessler (1994) and Raju and Meiburg (1995). This field continues to attract much interest to this day (see, e.g., Ravichandran et al., 2014 and references therein).

As additional examples deserving attention, Maxey et al. (1997) and Saw et al. (2008) reported numerical and experimental evidence, respectively, of spatial clustering of dense particles in highReynolds-number homogenous, isotropic turbulence. The formation of network-like regions of high particle density, characterized by a length scale depending on the Stokes number was also numerically predicted by Boffetta and De Lillo (2004). The common and current understanding of this long known but remarkable phenomenon of preferential concentrations relies on the idea that in a turbulent flow vortices can act as "centrifuges" ejecting particles heavier than the fluid and entrapping lighter ones (they create a positive divergence in the particle velocity field by ejecting them from their centers; Biferale et al., 2005; Chen et al., 2006; Bec et al., 2007).

Similar studies have been appearing for the specific problem of planetary formation. For a number of reasons, the flow in protoplanetary accretion disks is expected to be turbulent (Gammie, 1999 and references therein). Thermal convection has been suggested by several authors as one of the possible root causes (Cameron, 1978; Lin and Papaloizou, 1980; Ruden et al., 1988; Cabot and Pollack, 1992; Kley et al., 1993; Rozyczka et al., 1994). Another source of flow perturbation is shear-driven turbulence (Lynden-Bell and Pringle, 1974; Cuzzi et al., 1993).

Related numerical investigations have revealed that for small values of the particle Stokes number heavy dust particles rapidly concentrate in convergence zone between eddies in Kolmogorov-type homogeneous turbulence (Dobrovolskis et al., 1993) or, by contrast, in the cores of anticyclonic vortices in the solar nebula (Barge and Sommeria, 1995). For the latter case, it is also worth citing (Adams and Watkins, 1995; Tanga et al. 1996; Bracco et al., 1999; Godon and Livio, 1999; Barranco and Marcus, 2000), who were able to demonstrate (under different perspectives and in the framework of distinct approaches) that the local change in the Keplerian velocity of the disk caused 
by the anticyclonic motion in the vortex can induce a net (Coriolis) force toward the center of the vortex (the inverse happening in a cyclonic vortex).

Analytical results (Adams and Watkins 1995, using a geostrophic approximation) and stability analyses (see, e.g., Balmforth and Spiegel 1995 and Lovelace et al., 1999) have shown that many different types of fluid-dynamic disturbances and vortex solutions are possible in circumstellar disks.

It is also well known from experiment and simulation (consistent with theory) that for turbulent flows, in general, both the large and small scales of the turbulence can be important for particle transport (in several circumstances in nature and technology); indeed, while the large scales are generally responsible for diffusion phenomena (one-particle dispersion, with its dependence on gravitational settling and crossing trajectories), the small (Kolmogorov viscous dissipating) scales can be responsible for inter-particle collisions.

Apart from the abovementioned particle centrifugation, which occurs as a "local" phenomenon for relatively small values of the Stokes numbers, for greater values of this parameter the mechanism of segregation depends upon the entire history of an elemental volume of particles through the velocity field. The change of particle concentration along a particle trajectory is a multiplicative process leading to a log-normal distribution for the concentration of particles. Furthermore this behaviour can produce "caustics" or filamental structures where the particle concentration continuously increases (as the particles get compressed into smaller and smaller volumes). The particles within these filaments move in opposite directions. So in these regions the collision process is greatly enhanced not only because of high concentrations but of the higher relative velocities between particles (in other words, clustering enhances the neighbouring concentration of particles about any given particle and the velocity decorrelation between particles causes two nearby particles to collide, Sundaram and Collins, 1997). As the particles cluster onto a network of caustic lines (analogous to the networks of optical caustics that can be seen on the bottom of a swimming pool) this process can facilitate coagulation of particles by bringing them into close proximity, even in cases where the trajectories do not coalesce (Wilkinson and Mehlig, 2005; Wilkinson et al., 2007). This model of particle segregation in homogeneous isotropic turbulence was confirmed by Meneguz and Reeks (2011), who, among other things identified an interesting threshold value for the particle Stokes number above which the net compressibility of the particle concentration changes sign (from compression to dilation). All these processes are important influences on particle agglomeration and play a crucial role in (terrestrial) atmospheric phenomena such as droplet formation in turbulent clouds (Ayala et al., 2008; Wang et al., 2008; Devenish et al., 2012).

In the present work we focus on new categories of particle accumulation phenomena, discovered by very recent microgravity-related research, which seem to escape a possible simple definition or classification in the frame of the abovementioned main lines of research (still retaining, however, some affinities with the typical features and salient ingredients pertaining to such categories).

For illustration purposes, we use experimental results (as available) in synergy with companion numerical simulations. 
Insights are sought first from consideration of typical archetypal settings for microgravity conditions. More precisely, in Section 3, we describe the ability of inertia in promoting particle segregation and ordered accumulation when the background flow has the characteristics of the typical flow in well-known models of the floating zone (crystal growth) process in space (namely, the socalled liquid bridge). Although this should be regarded as a classical subject in the field of materials science, the universal character of the waveforms developing in the related supercritical state makes the conclusions applicable to a variety of phenomena going far beyond the specific technological problem considered. Similarly, Section 4 is entirely focused on particle attractors whose existence is made possible by the delicate interplay established between inertial phenomena and "alternating" vortical flows due to periodic "forcing" (still in the case of microgravity and noninterfering particles). The role played by inter-particle forces of various kinds is addressed in Section 5, with the specific case of aggregates induced by electrostatic forces being illustrated via "ad hoc" conceived experiments, executed in space or on the ground (with neutrally buoyant solid particles). Finally, through a critical assessment of the topology and morphology of the resulting structures (which appear in the form of one-dimensional circuits formed by aligned particles, twodimensional accumulation surfaces, three-dimensional compact structures, or fractal aggregates), we discuss a possible classification of the related particle attractors according to the prevailing effect.

A mathematical basis for the study of these problems is the theory of pattern formation in non-linear systems (this theory, in its broadest sense, attempts to classify and characterize the properties of all solutions of dissipative systems). Some researchers modeled some of these behaviours as the patterns formed by solid matter were controlled by one or more "attractors" (and it is known that more complex patterns, such as fractals, are formed by strange attractors; Lappa, 2009). However, in the framework of a less ambitious approach, also direct numerical discretization and solution of the system model equations can provide significant information about these topics for the case of dilute systems. Such equations include the Navier-Stokes equations in their complete form together with the energy equation (also simply referred to as thermal-convection equations) and a separate Lagrangian tracking scheme for the particles, based on the so-called Maxey-Riley equation or related variants or extensions (a flexible approach to account for a variety of forces potentially acting on transported particles, among them: the force exerted by the undisturbed flow, the buoyancy, the Stokes drag, the so-called virtual-added mass, the Basset "history" force, and potentially also time-varying density effects, the reader being referred, e.g., to Maxey and Riley, 1983 and Parmar et al., 2012 for additional details).

A similar degree of complexity is involved in the analytic or numerical treatment of the dynamics induced by particles interaction in concentrated systems. This problem requires adequate modeling of the related forces and approaches similar (form a theoretical standpoint) to those used for the Nbody problem, where each particle is allowed in principle to exert an influence on any other particle if it is located within a given ("cutoff") distance. 
Owing to the elaborate nature of some of these studies, it has not always been possible to fit an adequate account of them into the framework of the present survey, but attempts are made to give some indication of the most important results and of the methods employed.

\section{Accumulation Structures induced by Travelling Waves, Wave-Vortex and Wave-to-Wave Interactions}

Travelling waves are ubiquitous phenomena. They arise everywhere in nature and a number of technological processes involve their formation at some stage. They are the typical outcome of fluid-dynamic instabilities in Taylor-Couette and swirling flows (see, e.g., Schouveiler et al., 1999; Moisy et al., 2004; Abshagen et al., 2008), buoyancy (thermogravitational) convection (Clever and Busse, 1981; Hoyas et al., 2004; Lappa, 2016), surface-tension-driven (thermocapillary) convection (Preisser et al., 1983; Schwabe et al., 1992), baroclinic flows (Hide and Mason, 1975; Fein and Pfeffer, 1976), atmospheric convection (Rossby, 1940; Rhines, 1975) and many other circumstances (too many to be cited here).

Such disturbances are known (Lappa 2009, 2012) to have an impact on the resulting dynamics of a number of systems, ranging, e.g., from typical configurations used for materials processing (Lan, et al., 2000; Jing et al., 2004; Cröll et al., 1998), to the dynamics of complex atmospheric systems (Rhines, 1975) and even protoplanetary nebule (Goodman, 1993; Balmforth and Spiegel, 1995, Balbus and Hawley, 1998; Godon and Livio,1999; Bracco et al., 1999; Sheehan et al., 1999). Such waves can be isolated or undergo fascinating wave-to-wave interactions leading to more complex scenarios (Longuet-Higgins and Gill, 1967; Kuhlmann and Rath, 1993; Garnier et al., 2003; Williams, 2003; Abshagen et al., 2005; Shi and Imaishi, 2006, etc.).

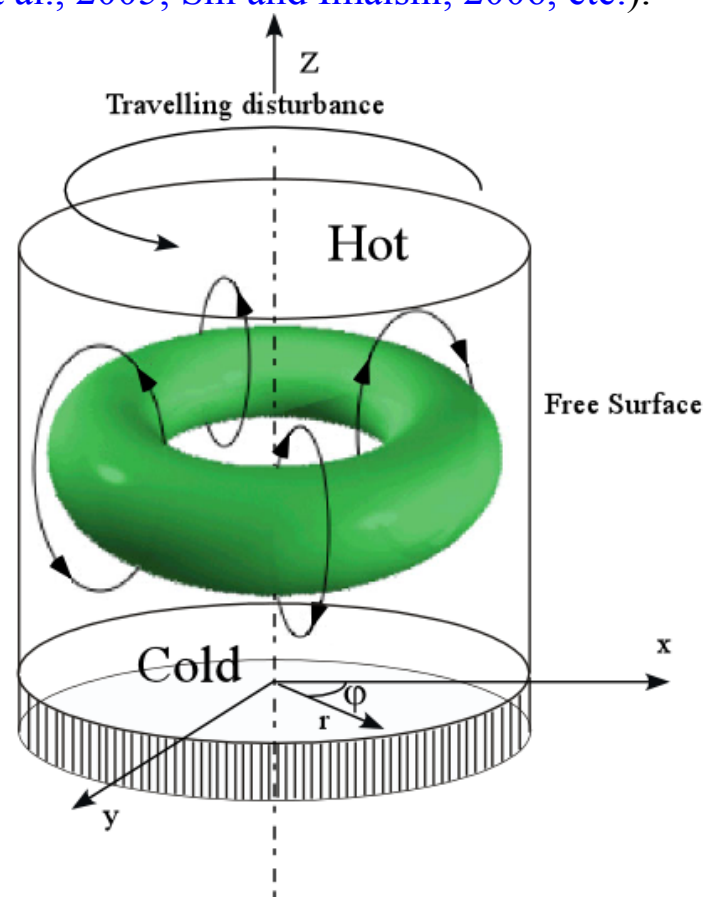

Figure 1: Sketch of the classical liquid bridge delimited by a cylindrical liquid-gas interface and held between two supporting disks at different temperatures. 
Without loss of generality, in this section we concentrate on the popular liquid-bridge problem (a floating column of liquid held between two cylindrical solid supports or disks, generally used as a simplified model of the Floating Zone technique (Lappa, 2003) for crystal growth from the melt, Fig. 1), which so much interest has attracted over recent years as a paradigm for the study of the fundamental properties of surface-tension driven flows, their stability and bifurcations in microgravity conditions.

By extensive past experimental investigation with transparent liquids (Shevtsova et al., 2011), it is known that for sufficiently small values of the Marangoni number (eq. (11)), the resulting (thermocapillary) convection is laminar, steady and axisymmetric (a steady vortex ring is established inside the liquid bridge in which the rotating fluid takes on a toroid or doughnut shape; this ring consists of surface flow moving from the hot side to the cold side of the outer surface and a return flow in the interior of the liquid bridge). When this characteristic number exceeds a certain critical value depending on the Prandtl number of the liquid, on the geometry (height to diameter ratio $\mathrm{A}$ ) and on the thermal boundary conditions, the flow undergoes a transition to a complex oscillatory three-dimensional (3D) pattern characterized by the propagation of waves in the azimuthal direction.

More recent studies have revealed that, in such circumstances, some spatial paths can be identified which tend to "capture" (as time passes) rigid particles seeded in the liquid (in general, tracers, which are injected in the liquid for visualization purposes). A first (albeit partial) observation of this behaviour in liquid bridges is due to Schwabe et al. (1996).
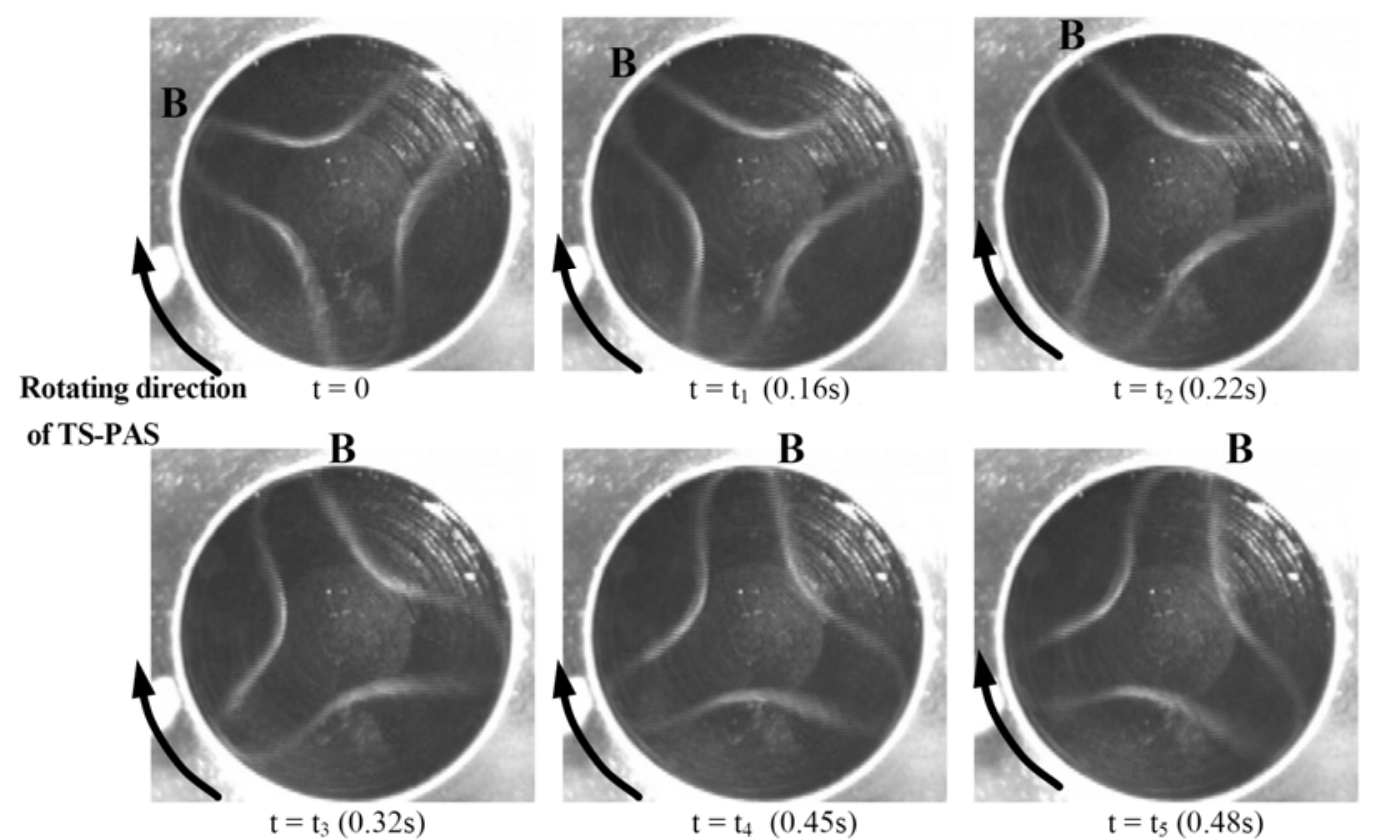

Figure 2: Particle accumulation structures (view through a top transparent disk made of glass) for a 2 cSt silicone oil liquid bridge with aspect ratio (height/diameter) $\mathrm{A}=0.32, \mathrm{Ma} \cong 3.1 \times 10^{4}$ $\left(\mathrm{Ma}=\sigma_{\mathrm{T}} \Delta \mathrm{TL} / \mu \alpha\right.$ where $\mathrm{L}$ is the bridge height; if one looks at the full dynamics, the closed string formed by particles is rotating in the azimuthal direction as a unit; experimental results courtesy of I. Ueno). 
The distinguishing feature of this new category of attractors with respect to other flows, is the strictly "one-dimensional nature" (1D) of the structures formed by particle accumulation. All particles tend to accumulate as time passes along a closed circuit moving "collectively" such that an illusion of a solid item, rotating at the same angular velocity of the wave, is created (such structures appear as seemingly rigid filaments, rotating without changing their shape, Fig. 2). This has opened the problem to discern whether the related dynamics can be still explained in the framework of inertial models, like those used to justify the preferential concentration of particles, drops or bubbles in turbulent flows (although not in strictly 1D structures), as discussed earlier, or other specific noninertial models have to be necessarily invoked.

A structure is formed because there is a large number of particles performing spatially correlated large-scale motions. The reader is referred to, e.g., Kuhlmann et al. (2014), for a recent review of a large cross section of fundamental research on this topic. Here we limit ourselves to recalling that the phenomenon was placed in a more relevant theoretical context by Pushkin et al. (2011), who addressed the role played by particle inertia and introduced the concept of "phase locking" (alternatively called "frequency locking or entrainment", this fundamental phenomenon is known to emerge in systems with a weak interaction between modes with close frequencies). More precisely, the model was based on the idea that (as also originally argued by Schwabe et al., 2007) the turnover motion of a particle around the toroidal roll of the underlying Marangoni flow may tend to become "synchronized" with the fluid-dynamic oscillations produced by the rotating wave, thereby supporting particle self-assembly.

A later re-visitation of this model in terms of particle axial vorticity is due to Lappa (2013b), who replaced the particle turnover frequency with equivalent arguments on the particle angular spin.

By introducing the two components of vorticity $\underline{\zeta}=\underline{\nabla} \wedge \underline{V}$ in the azimuthal $(\varphi)$, and axial (z) directions as

$$
\begin{aligned}
& \zeta_{\varphi}=\left(\frac{\partial V_{r}}{\partial z}-\frac{\partial V_{z}}{\partial r}\right) \\
& \zeta_{z}=\frac{1}{r}\left[\frac{\partial}{\partial r}\left(r V_{\varphi}\right)-\frac{\partial V_{r}}{\partial \varphi}\right]
\end{aligned}
$$

(where $V_{z}, V_{r}$ and $V_{\varphi}$ are the fluid velocity components along $z, r$ and $\varphi$, respectively, see Fig. 1), Lappa (2013b) used such quantities to re-interpret the phenomenon of particle accumulation from a vorticity-based perspective.

Along these lines, it is worth recalling that these two components should be regarded, respectively, as a measure of the strength of the basic toroidal roll $\left(\zeta_{\varphi}\right)$ and departure from the axisymmetric state $\left(\zeta_{\mathrm{z}}\right)$. The latter contribution, in fact, is zero if it is evaluated in the axisymmetric (subcritical) state, but it is nonzero in a $3 \mathrm{D}$ state where, more specifically, its half $\left(\zeta_{z} / 2\right)$ can be regarded as a measure of the local average angular velocity (spin) of the considered fluid element about the vertical direction (hereafter for simplicity it will be referred to as $\Theta_{\text {fluid }}$ or simply $\Theta$ ). 
By determining the supercritical state of the Marangoni flow in the liquid bridge by direct numerical solution of the Navier-Stokes and energy equations in their complete, time-dependent and non-linear formulation (Fig. 3a) and computing, at the same time, the motion of dispersed particles by solution of the well-known Maxey-Riley equation, specific insights into the ordered accumulation of particles were obtained by plotting particles locations in space combined with the isosurfaces of $1 \frac{1}{2}$ of the axial component of vorticity $\left(\Theta_{\text {fluid, }}\right.$ Fig. $\left.3 b\right)$.
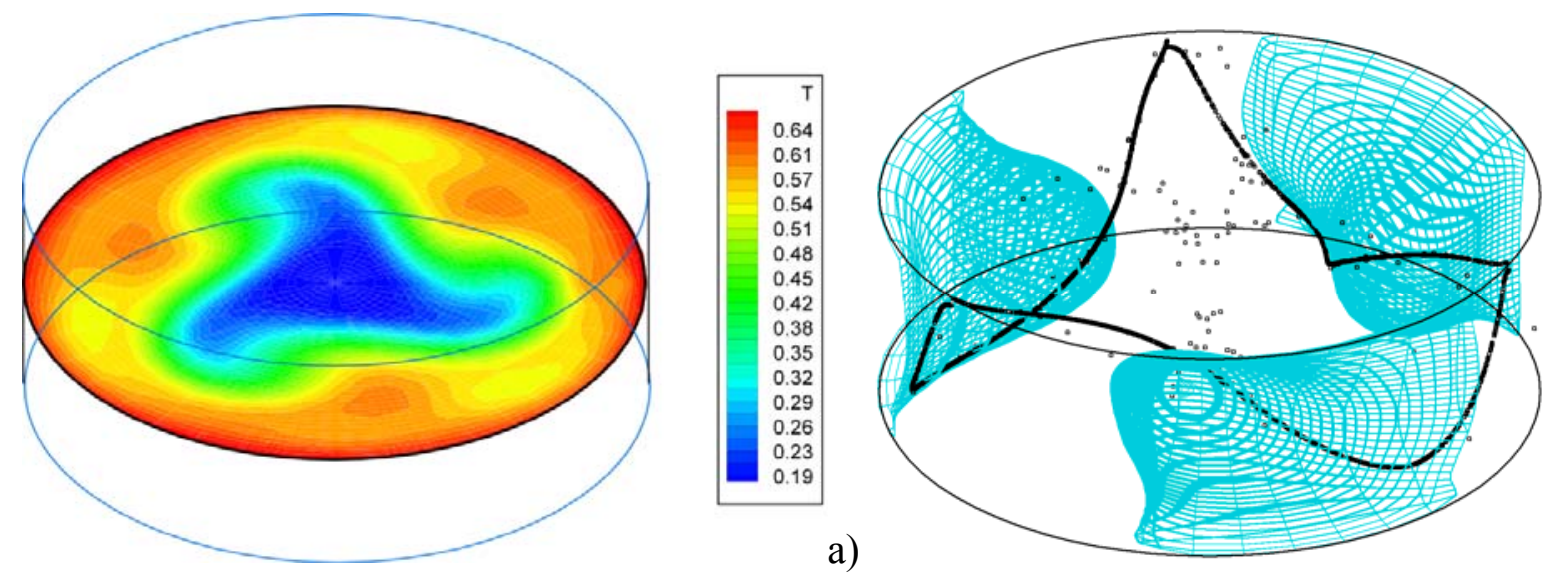

b)

Figure 3: Liquid bridge $\left(\operatorname{Pr}=8, \mathrm{~A}=\mathrm{L} / \mathrm{D}=0.34, \mathrm{Ma} \cong 2.1 \times 10^{4}, \mathrm{Ra}=0\right.$, azimuthal wavenumber $\mathrm{m}=3$, $\omega_{\text {wave }}=71.4$ ): a) Snapshot of travelling wave state (temperature contour map at midheight between the supporting disks); b), Particle accumulation structures together with isosurfaces of fluid angular spin plotted for $\Theta_{\text {fluid }}=72, \xi=1.85, \eta=1 \times 10^{-5}$.

The wave was allowed to develop and travel through a domain initially seeded with a uniform collection of particles. By this approach, a precise relationship between the emerging onedimensional structures (closed particle-dense lines) and the two-dimensional (2D) isosurfaces of $\Theta_{\text {fluid }}=\zeta_{z} / 2$ could be identified. More precisely, evidence was provided that particle structures tend to "stay attached" for most of their azimuthal extension to the isosurfaces of fluid axial angular velocity $\Theta_{\text {fluid }}\left(=\zeta_{z} / 2\right)$ such that $\Theta_{\text {fluid }}=\omega_{\text {wave }}$ (where $\omega_{\text {wave }}$ is the angular frequency of the travelling hydrothermal wave). It was shown that the spatial shape and curvature of the isosurfaces does not determine solely the one-dimensional pattern created by the projection of the particle circuit in the $\mathrm{xy}$ plane; but it also drives the local inclination (with respect to the $\mathrm{z}$ axis or equivalently with respect to the horizontal boundaries) of such structures in the 3D space (Fig. 3b).

In this way, the so-called inertial theory for explaining these dynamics was validated and extended phenomenologically (via numerical simulation) by incorporating ideas of vorticity-wave interactions (leading to cast the equality $\Theta_{\text {fluid }}=\omega_{\text {wave }}$ as a "practical implementation" of the necessary condition initially theorized by Pushkin et al., 2011).

Later studies have demonstrated that such one-dimensional paths attracting particles in the physical space are neither an exclusive prerogative of Marangoni convection nor an exclusive feature of systems with a free liquid/gas interface, their occurrence being possible also in other circumstances (provided specific conditions are established or satisfied). 


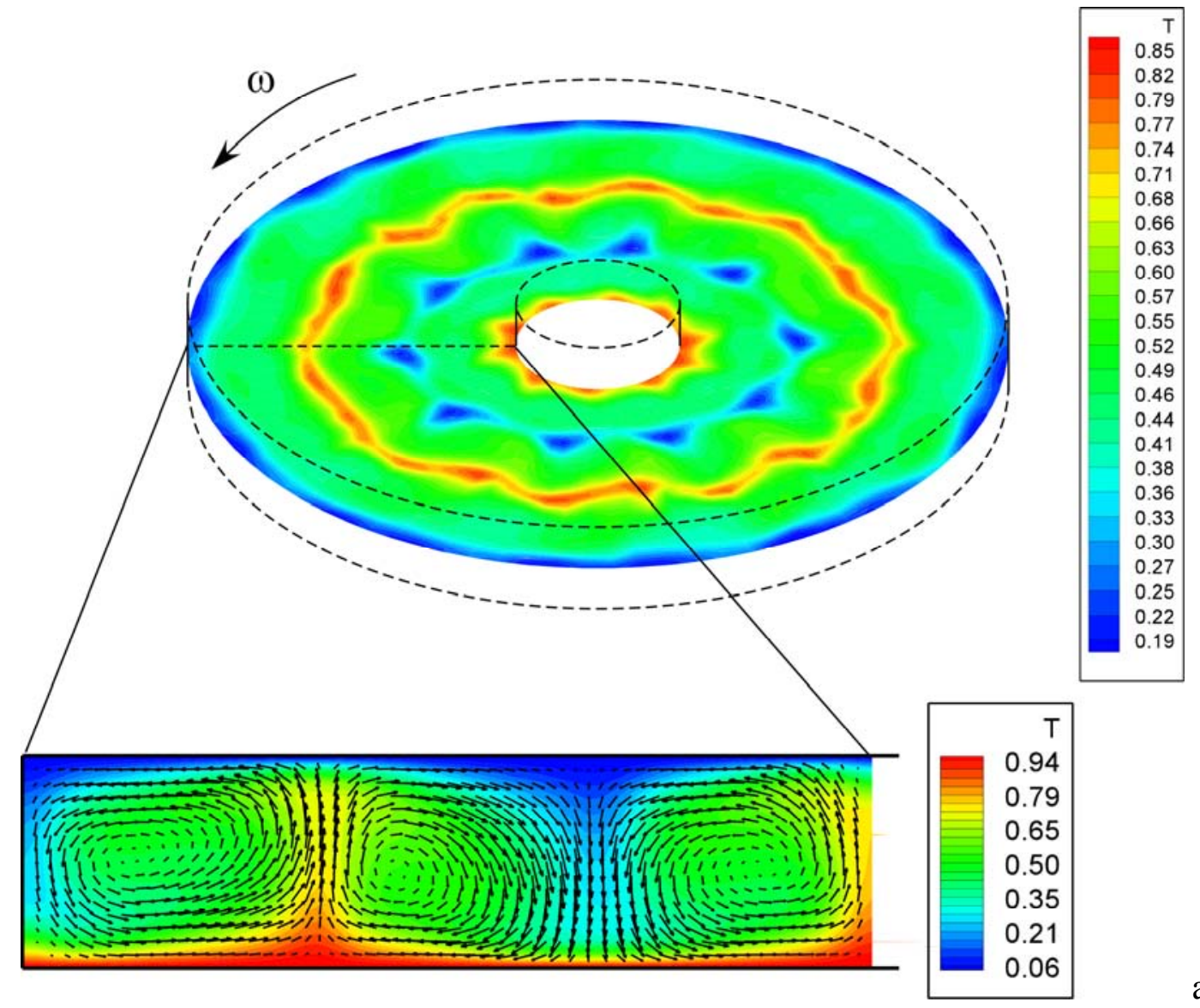

a)

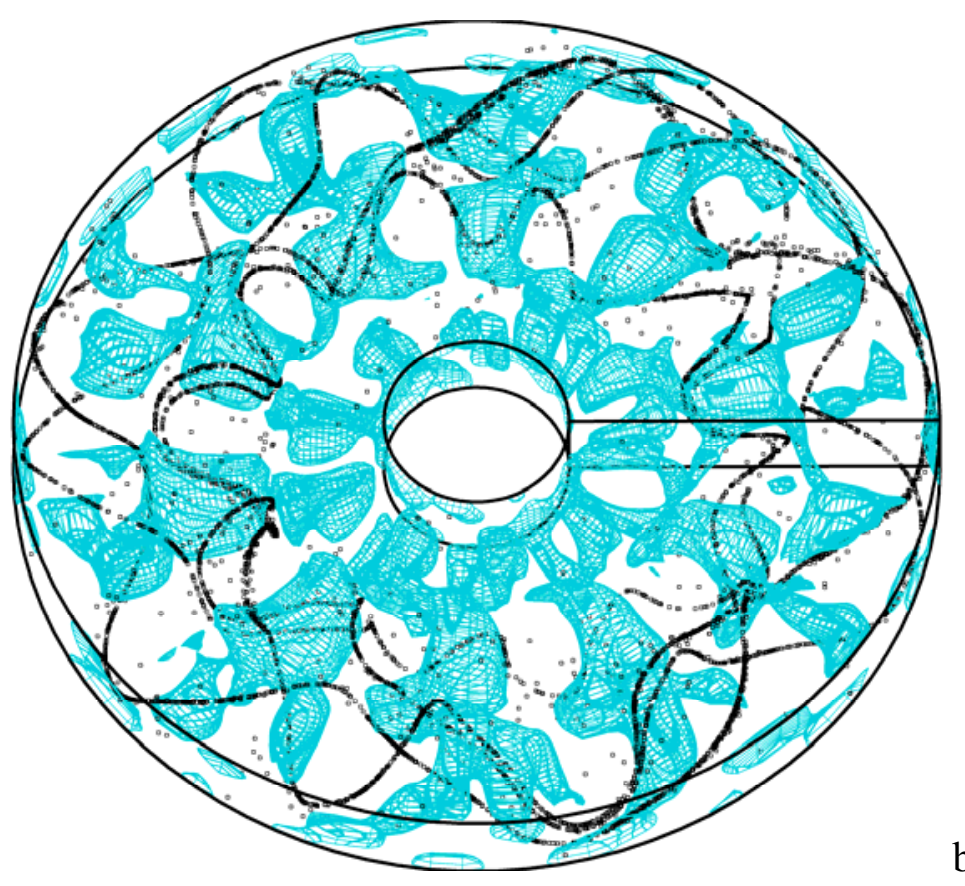

b)

Figure 4: a) Snapshot of travelling-wave state of $\mathrm{RB}$ convection for $\mathrm{Pr}=1$, and $\mathrm{Ra}=3.5 \times 10^{4}$ (temperature distribution for $\mathrm{z}=0.5$ and related thermofluid-dynamic field in a meridian section for normal gravity conditions); b) Particle lines and isosurfaces of fluid (local) angular velocity for $\Theta_{\text {fluid }} \cong 5.4$ and $\eta=8.5 \times 10^{-5}$ (the individual particles organize themselves into what appear to be rotating solid threads, these particle-dense lines stay attached to the isosurfaces of fluid local angular velocity where $\Theta_{\text {fluid }}=\Omega$ as predicted by the so-called phase-locking theory). 
Indeed, it was shown that one-dimensional sets formed by the accumulation of tracer particles can also emerge in flows of thermogravitational nature (Rayleigh-Bénard convection) in the region of the space of parameters, in which the so-called OS (oscillatory solution) flow of the Busse balloon (yet a travelling wave) represents the dominant secondary mode of convection (Lappa (2013c, Fig. 4).

It was confirmed that, if the $\Theta_{\text {fluid }}=\omega_{\text {wave }}$ condition is satisfied somewhere in the velocity field, particles, initially uniformly spaced in the liquid, are allowed to demix and form "apparently solid threads", which rotate at an angular velocity equal to the angular frequency of the thermofluiddynamic disturbance travelling in the azimuthal direction (as shown in Fig. 4b, a strong geometrical correlation can be identified again between the isosurfaces where $\Theta_{\text {fluid }}=\omega_{\text {wave }}$ and the emerging one-dimensional particle structures).

Over the subsequent years, additional studies trying to address the bizarre phenomena which emerge when the considered flow has the form of a standing wave (Lappa, 2014a), have provided further evidence for the relevance of this class of inertial models to a variety of situations of technological or theoretical interest.

As a sample standing wave (SW) here we use again the typical supercritical thermocapillary (Marangoni) flow (both travelling waves and standing waves are known to be possible waveforms of the supercritical Marangoni flow, preference for one or the other state being dependent on the distance from the onset of instability). Our conclusions, however, are applicable to pulsating flows of gravitational nature as well and, in general, to any flow with the characteristics of a SW.

In physics, a standing wave (also known as a stationary wave), is a wave (a disturbance oscillating in time) that remains in a constant position. In a stationary medium this phenomenon generally emerges as a result of the interference (superposition) of two waves having the same amplitude and same angular frequency $\omega$, but travelling in opposite directions.

Assuming a general representation with each wave having an amplitude $B(\mathrm{r}, \mathrm{z})$ and an initial phase $G(\mathrm{r}, \mathrm{z})$ of the type $F_{ \pm}=B(r, z) \exp \{i[ \pm m \varphi-\omega t+G(r, z)]\}$ (where $F$ is a generic quantity such a temperature or a velocity component, with the symbol \pm indicating propagation in the clockwise or anticlockwise circumferential direction $\varphi$ ), by simple mathematical developments it is easy to verify that a superposition of two counterpropagating waves with the same amplitude should result simply in

$F=2 B(r, z) \cos (m \varphi) \cos (\omega t-G(r, z))$

Since the oscillatory term in this equation does not depend on $\varphi$, this situation represents in principle a waveform characterized by maximum and minimum disturbances fixed in space with the minimum being continually replaced by the maximum and vice versa (as soon as cos $(\omega t-G(r, z)$ ) changes its sign). As an example, in terms of temperature these extrema would correspond to hotter and colder zones in the physical domain, with the resulting threedimensional temperature 
disturbance simply consisting of a number $m$ of couples of spots (hot and cold) "pulsating" at the same azimuthal positions along the interface of the liquid bridge.

This typically results in a field with no net disturbance transport on average along the propagation direction of the two component waves (the azimuthal direction $\varphi$ in the present case for which we refer to a liquid bridge). In terms of patterning behaviour, the final effect is a series of nodes (disturbance zero amplitude) and anti-nodes (disturbance maximum amplitude) at fixed locations along $\varphi$ (as shown, e.g., by Lappa et al., 2001, the node/anti-node distance is $\lambda / 2$ where $\lambda=\pi / \mathrm{m}$ ).

While the center of these (hot or cold) spots (i.e. the "anti-node"), retains a fixed location in space, their azimuthal (and axial) extension changes in time. More precisely, the "front" (or external boundary) of these growing and shrinking spots can be "seen" as a disturbance originating at the anti-node and travelling outward or inward (along the azimuthal and axial dimensions) depending on whether the considered spot is in a growing or contracting stage, respectively. Albeit there is no net disturbance transport along the positive (or negative) azimuthal direction, the resulting disturbance apparently propagates from each anti-node to the two surrounding nodes with variable (location-depending) angular velocity. More specifically, by indicating with $\Omega$ the variable disturbance angular frequency, the following conditions represent a good model of a $\mathrm{SW}: \Omega=0$ on all nodes, $|\Omega|=\omega$ on all antinodes, with all points located in other meridian planes experiencing an apparent disturbance angular velocity $0<\Omega \leq \omega$ (Fig. 5a). This "alternate" view with respect to eq. (19) may be regarded as a more "phenomenological" representation of a standing wave in which the disturbances resulting from the superposition of two counterpropagating waves travel in the azimuthal (and axial) direction with a "variable" velocity. This particular perspective is at the root of the explanations elaborated by Lappa (2014a) to provide an interpretation of the related particle accumulation phenomena on the basis of the phase-locking mechanism (originally introduced, as discussed before, to explain the formation of 1D structures for the travelling wave case).

By direct experimental investigation, yet with the liquid bridge and its supercritical flow (see Schwabe and Mizev, 2011), it is known that when the flow is a standing wave, the patterns created by the spontaneous demixing and accumulation of particles show an appearance that is totally different from those formed by travelling waves. The essentially stationary nature of the resulting pattern as seen in the laboratory frame and its topological properties distinguish such a case from phenomena observed previously (for which particles were seen to collapse along a closed path looking like an m-blade "windmill" rotating at a constant speed, where $\mathrm{m}$ is the azimuthal wavenumber, see again Figs. 3 and 4).

From available experiments (Fig. 5a), one can see that the particle pattern consists of $2 \mathrm{~m}$ sectors (6 sectors for $\mathrm{m}=3$ ), which, like 6 pieces of a cake, are separated by 6 sharp radial stripes formed by close particles. The pattern also includes an inner particle circuit with shape and average radial position varying in time. 

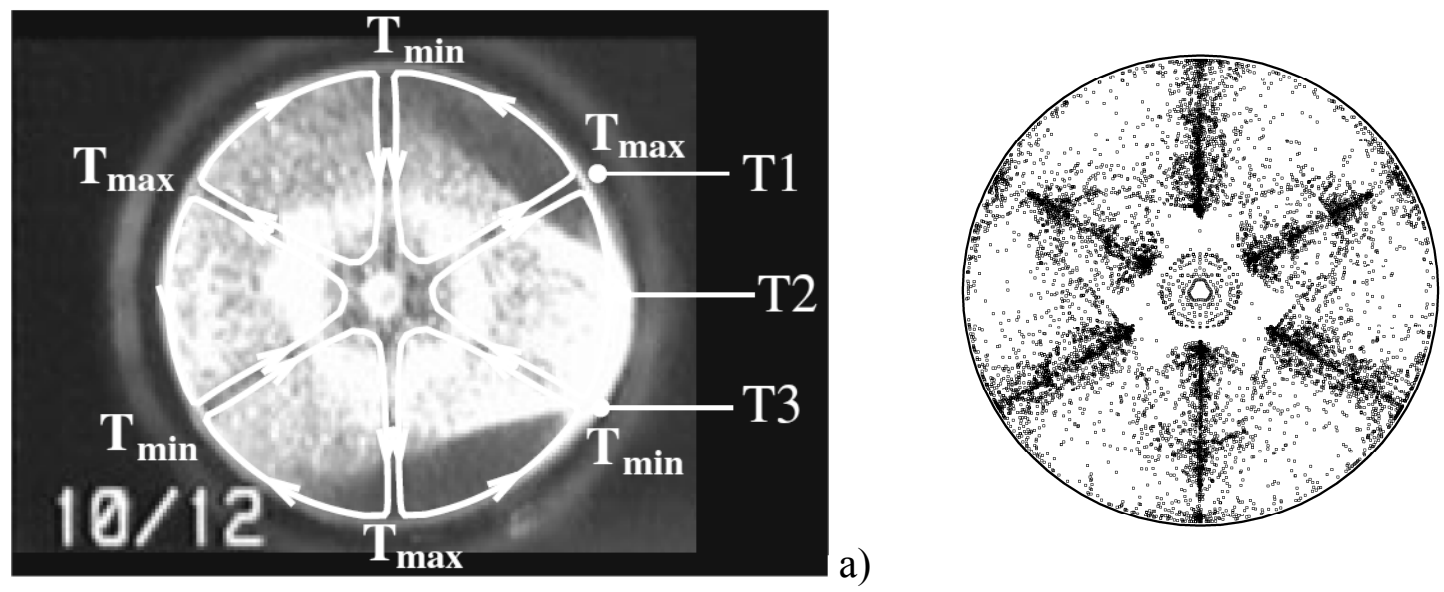

b)

Figure 5: Standing particle accumulation structures: a) projection in the xy plane of particle distribution (experimental results, snapshot, courtesy of D. Schwabe and A. I. Mizev); b) projection (snapshot) in the xy plane of the numerically-determined three-dimensional particle distribution for a liquid bridge of $\mathrm{NaNO}_{3}, \mathrm{Pr}=8, \mathrm{~A}=0.34, \mathrm{Ma} \cong 2.1 \times 10^{4}, \mathrm{Ra}=0$, standing wave, $\omega=71.4, \xi=1.85$, $\eta=1 \times 10^{-5}$.

These new intriguing dynamics, which can be explained neither in terms of stagnation surfaces (the aforementioned radial branches and the inner circuit are not stagnation loci), nor on the basis of KAM tori (given the absence in the considered flow of azimuthally extended closed stable streamtubes, whose existence is a necessary prerequisite of that class of models) can be given a relatively simple explanation if the problem is cast again in the form of axial vorticity dynamics and related physical connections with inertial mechanisms (Lappa, 2014a).

Towards this end, it is sufficient to consider the general physical consequences of the above representation of SW when it is considered in combination with the resonance (phase-locking) theory discussed before. Indeed, in the light of such a theory and related revisitation in terms of vorticity (hereafter, simply referred to as the vorticity-wave model), particles should accumulate where the angular spin

$\Theta_{\text {fluid }}=\frac{1}{2}[\underline{\nabla} \wedge \underline{V}]_{z}$

attains a value $\cong 0$. Any particle located at a position where $\Theta_{\text {fluid }} \neq 0$ and, in particular, $\Theta_{\text {fluid }} \cong \Omega$, in fact, would tend to undergo the vorticity-wave coupling/locking mechanism and, as a result, to leave its initial position (moving in the azimuthal direction by virtue of the resonance (or synchronization) physical process described before.

A natural consequence of the above arguments is that particles accumulate in the six meridian planes shown in Fig. 5 because those are the only locations where the vorticity goes to zero ( $\Theta_{\text {fluid }} \cong 0$, if the particles leave such locations they are immediately pushed back to such planes by the abovementioned phase-locking process). Such arguments are fully supported by results obtained by means of numerical simulation (Fig. 6). 

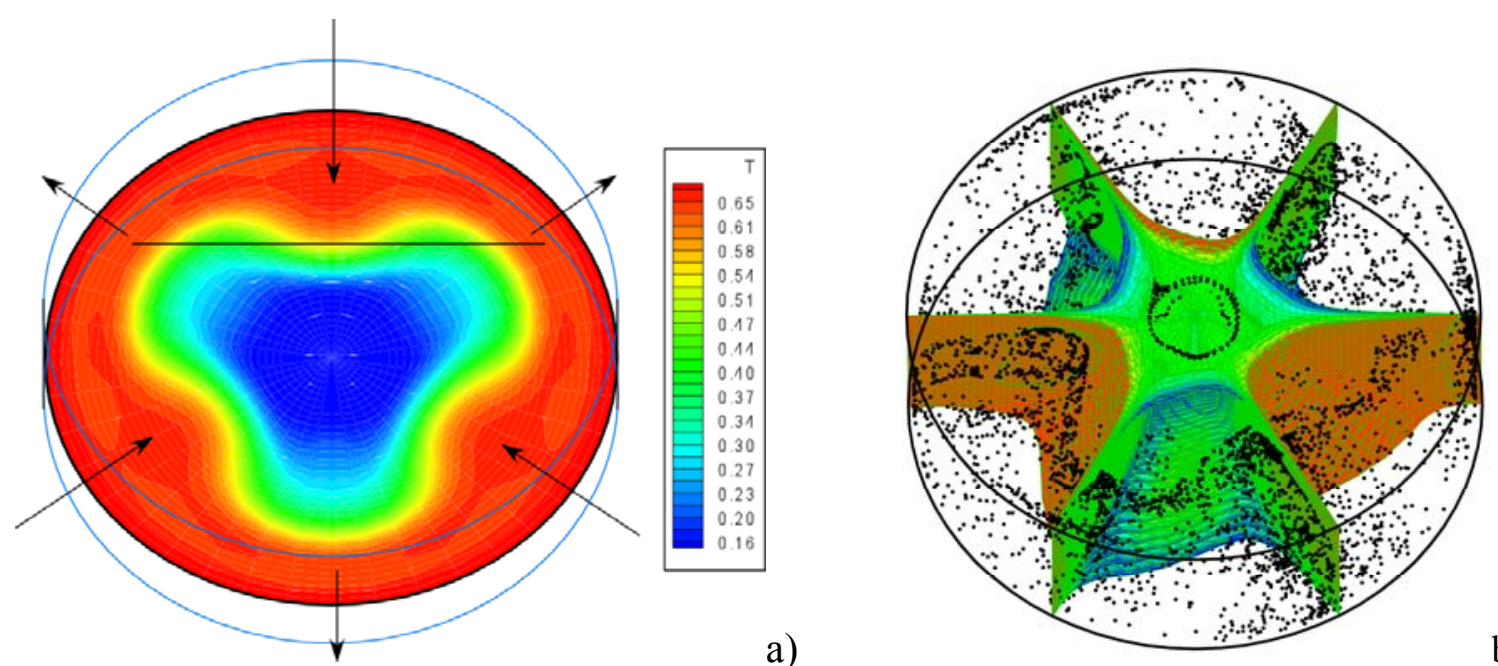

a)

b)

Figure 6: liquid bridge of $\mathrm{NaNO}_{3}, \mathrm{Pr}=8, \mathrm{~A}=0.34, \mathrm{Ma}=20600, \mathrm{Ra}=0$, standing wave, $\omega=71.4$, $\xi=1.85, \eta=1 \times 10^{-5}$ : a) Snapshot of the standing wave state (temperature contour map at midheight between the disks, the arrows are used to indicate the directions along which the pattern periodically expands and retreats); b) 3D snapshots of particle distribution and isosurfaces of $\Theta_{\text {fluid }} \cong 0$ ($\left.1 \leq \Theta_{\text {fluid }} \leq 1\right)$.

Stripped to its basics, the related explanation for particle structure formation envisions a restoring effect played by the vorticity-wave coupling mechanism in keeping particles confined to specific zones of the physical space, in particular, those where particles cannot phase lock with disturbances locally travelling in the azimuthal direction.

Given the general nature of these phenomena, not dependent on the specific properties of the surface-tension-driven flow (as demonstrated by their occurrence in flows of gravitational nature), it can be speculated (see, e.g., Schwabe and Frank, 1999) that these mechanisms might play a significant role as well in problems of astrophysical interest such as planet formation from protoplanetary nebule (the only necessary prerequisite being the existence of some background vorticity and travelling fluid-dynamic disturbances).

The robustness of particle clustering induced by wave propagation suggests that it could be a ubiquitous process. Moreover, both travelling waves and standing waves are known to be typical solutions of the protoplanetary disk stability problem (Goodman, 1993; Balmforth and Spiegel, 1995, Balbus and Hawley, 1998; Godon and Livio,1999; Bracco et al., 1999; Sheehan et al., 1999).

Obviously, the phase-locking mechanism might be in some way disturbed by the mutual forces or particle "collisions". It cannot be excluded, however, that circumstances might exist where this process becomes operative (especially on more "external" orbits of a solar system, where the density of dispersed solid matter is very small and collisions are less likely to occur; by contrast on such orbits the presence of gases is larger due to the pressure exerted on gas molecules by the solar wind, which tends to push them from the internal regions of the solar system outwards).

Before moving to the next subject, it is worth recalling here that inertia does still play a crucial role in such phenomena as witnessed by numerical simulations showing that when particles recover the motion of ideal Lagrangian tracers (in the limit of vanishing size and mass), they are prevented from undergoing self-assembly. For all the situations discussed in this section, such structures were 
found to emerge only if the inertial parameter $\eta$, defined by eq. (16) was larger than a threshold value (with recognizable particle patterns disappearing in the limit as $\eta \rightarrow 0$ ).

\section{Periodic Forcing}

Time-periodic flows are not necessarily a consequence of fluid-dynamic instabilities such as those discussed in the preceding sections for the case of thermocapillary or thermogravitational convection. There are other circumstances in which oscillatory flows supporting particle selfassembly can be produced by the application of an external time-varying forcing to the considered system. This is the typical case occurring, for instance, onboard the International Space Station, where a spectrum of accelerations at different frequencies is present, ranging from zero (steady residual acceleration) to hundreds of Hertz. These unsteady contributions to the residual gravity field, are generally separated into "quasi-steady", having a low frequencies content, as those due to atmospheric drag, solar radiation pressure, higher harmonics of the Earth gravitational field; and the so-called "g-jitters" having a higher frequencies content, as those coming from pulsating or impulsive external loads, like motor firings during manoeuvres, crew activity, station motorized equipments, station moving elements, space debris impacts, and the station structural elastic response to these loads (in the form of "vibrations").

Just as steady gravity on Earth can lead to the onset of buoyancy convection in the presence of a temperature gradient, a time-varying (periodic) residual acceleration can promote fluid convection in a non-isothermal fluid. This kind of convection, generally referred to as "thermovibrational flow" (see, e.g., Savino and Lappa, 2003; Lappa, 2015) has enjoyed special attention over recent years due to its perturbing (undesired) influence on a variety of experiments performed in microgravity conditions (Monti et al., 1998 and 2001).

In this section we focus on the action of alternating flows with a well-defined frequency on suspensions of solid matter through nonlinear effects. The specificity of the considered alternating flows is that their linear effects have zero-time averaged value. Hence, only nonlinear effects are expected to be significant even if they are small compared to the instantaneous linear effects. As already explained to a certain extent in Sect. 2, we assume such alternating flows to be produced by a displacement of the considered geometrical system along a given direction ( $\underline{\hat{n}}$ is the related unit vector) varying in time as $\underline{s}^{l a b}(t)=b \sin (\omega \mathrm{t}) \underline{\hat{n}}$ where $\mathrm{b}$ is the displacement amplitude and $\omega=2 \pi f$ is the related angular frequency, this leading to an acceleration $\underline{g}(t)=g_{\omega} \sin (\omega \mathrm{t}) \underline{\hat{n}}$ where $g_{\omega}=\mathrm{b} \omega^{2}$ (we introduce the related nondimensional acceleration amplitude as $\gamma=b \omega^{2} / g$ where $\mathrm{g}$ is the standard gravity acceleration at the sea level, i.e. $g=9.81 \mathrm{~ms}^{-2}$ ).

Such inertial perturbations are known to act on "inhomogeineities" present in the considered fluid system. These inhomogeineities can be due (see, e.g., Carotento and Lappa, 2003) to the granular structure of matter itself, as an assembly of hard particles, or to density inhomogeineities, as those induced in a simple fluid by temperature gradients (which lead to the aforementioned thermovibrational flow). 
Along these lines of inquiry, most recently, a completely new phenomenon of particle accumulation in vibrated monodisperse suspensions of solid spheres (in a non-isothermal liquid) has been identified.

Evidence has been provided that even in situations in which particle-particle hydrodynamic interactions are negligible (dilute systems), intriguing non-linear effects can lead to the irreversible formation of well-defined particulate structures over "long" temporal scales, i.e. times much larger than the period of the applied vibrations. The long-range translational ordering is produced by the delicate interplay between convective effects (of thermovibrational nature) and the (inertial) response of each isolated particle to the time-periodic acceleration (the related mechanism envisions a cooperative action exerted on particles by inertial forces and vorticity of thermovibrational nature, as demonstrated by the fact that the formation of well-defined structures is prevented when one of these two effects is not taken into account, Lappa, 2014b).

Such vorticity is generated essentially by the interplay between the temperature distribution and the instantaneous acceleration caused by vibrations. More precisely, such acceleration and the imposed temperature difference alternatively cooperate to produce recognizable clockwise or anticlockwise convective rolls in their plane over a cycle of modulation.

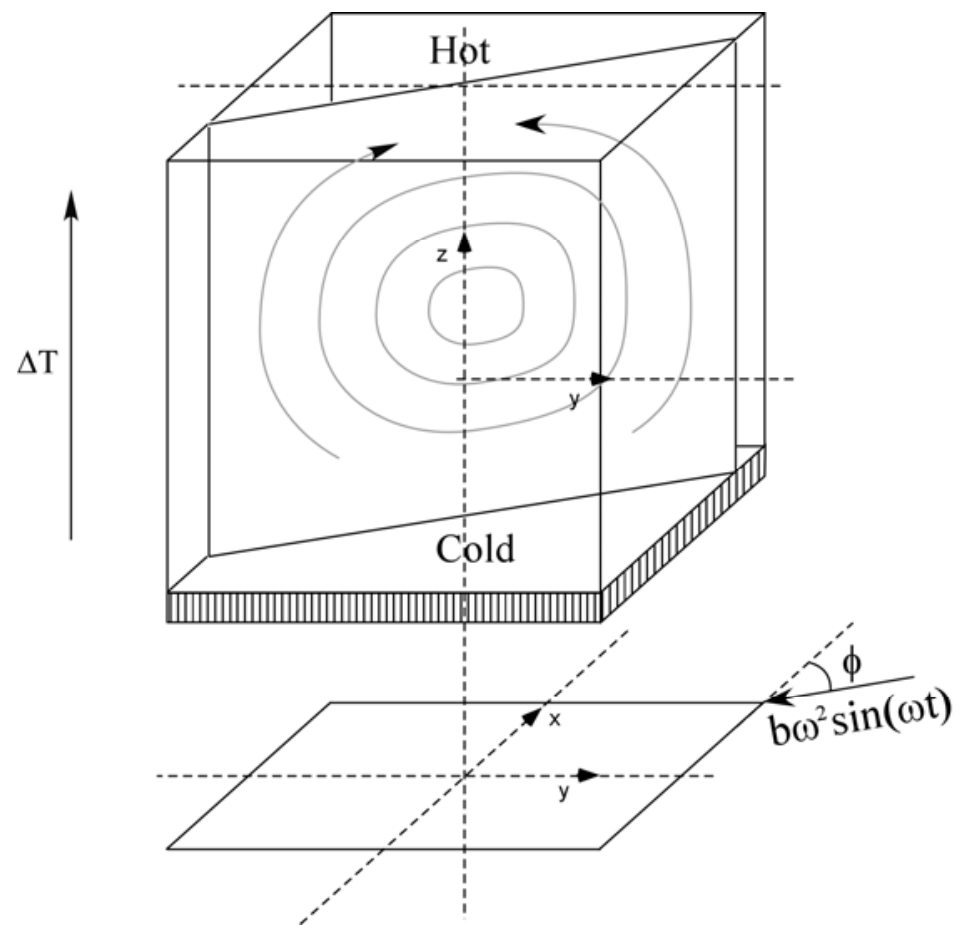

Figure 7: Cubic cavity with characteristic size L shown, delimited by solid walls (one at $\mathrm{z}=-0.5$ cooled, the other at $\mathrm{z}=0.5$ heated, adiabatic conditions on the remaining sidewalls). The vibrations are contained in the $\mathrm{xy}$ plane and form an angle $\phi$ with the $\mathrm{x}$ axis $(0 \leq \phi \leq \pi / 4)$.

In the interest of conciseness, the examples illustrated in the following are limited to the system sketched in Fig. 7 (a cubic cavity containing a fluid and dispersed particles, subjected to an imposed temperature difference and vibrations perpendicular to such a gradient). 
As for the simulations discussed in Sect 3, this computational domain has been seeded initially with a monodisperse collection of stationary spherical particles.

As shown in Fig. 8, even for such a simple geometry, a striking feature of the emerging patterns is their multiplicity. A variety of accumulation structures is possible according to the relative direction of vibrations with respect to the walls. When the angle $\phi$ shown in Fig. 7 increases from 0 to $\pi / 4$, the shape of the accumulation region evolves from a cylindrical surface to compact surfaces (a kind of irregular ovoids) passing through the intermediate stage of a conical surface, which might be the signature of some hidden analytic order underlying the formation and existence of such attractors in the physical space (Lappa, 2014b).

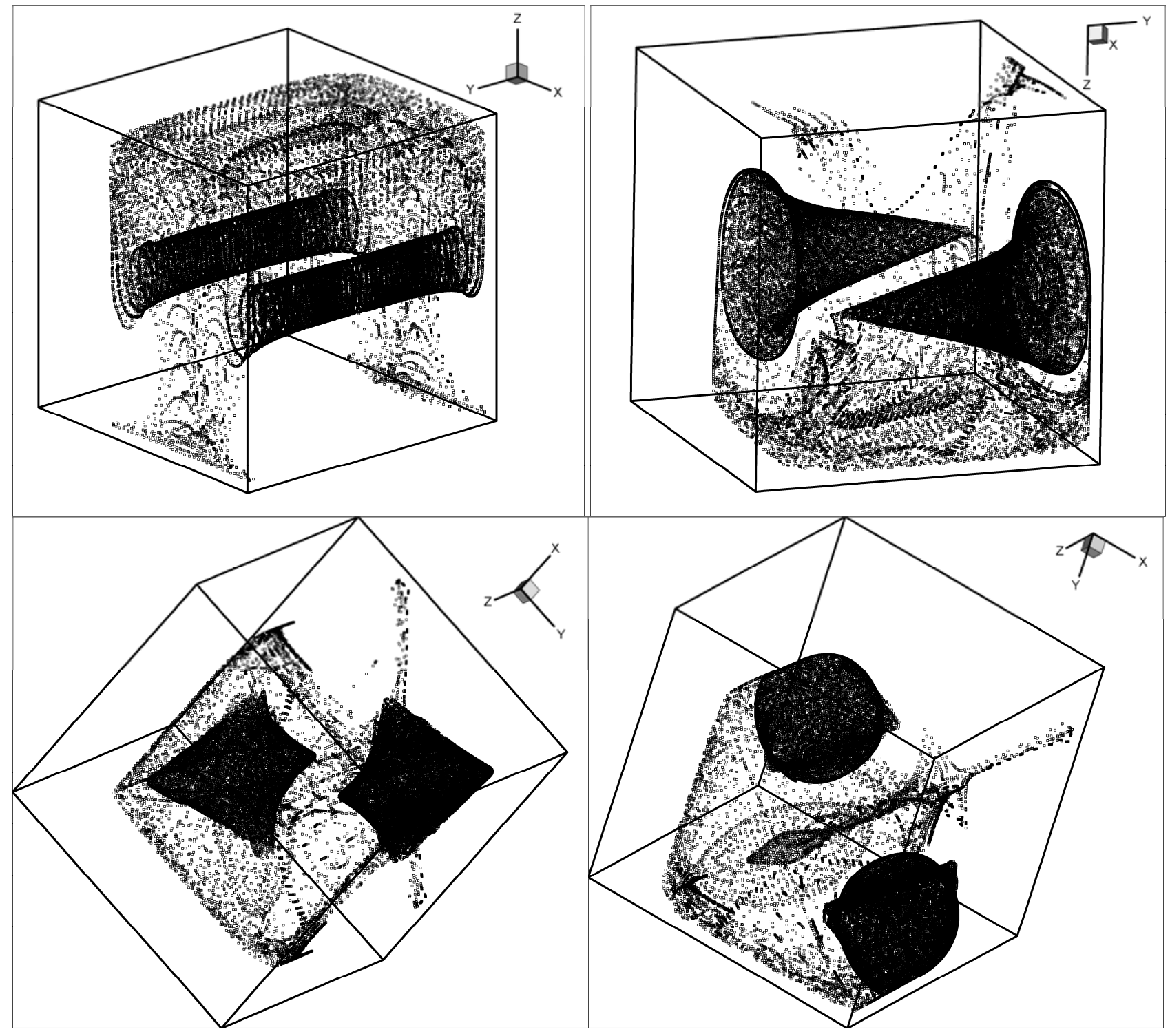

Figure 8: Particle aggregates (3D view) for $\varpi=1 \times 10^{3}, \gamma=b \omega^{2} / g=3 \times 10^{5}, \operatorname{Pr}=8, \operatorname{Ra}=0$, $\operatorname{Ra}_{\omega}=1.58 \times 10^{4}, \xi=1.85$, and $\eta=1 \times 10^{-4}$ (no steady gravity) a) $\phi=0, b$ ) and c) $\phi=\pi / 4, d$ ) $\phi=\pi / 8$. 
Under the effect of vibrations the final pattern oscillates back and forth along the direction of imposed vibrations apparently as a whole at the vibration frequency as experienced by an observer in the laboratory (fixed) reference frame.

Further insights into this phenomenon can be obtained via a parametric analysis of its dependence on the typical problem parameters (vibration frequency and particle characteristic size). The strong dependence of the formation time on the parameter $\eta$ (Fig. 9), indeed, provides evidence for its "inertial" nature.

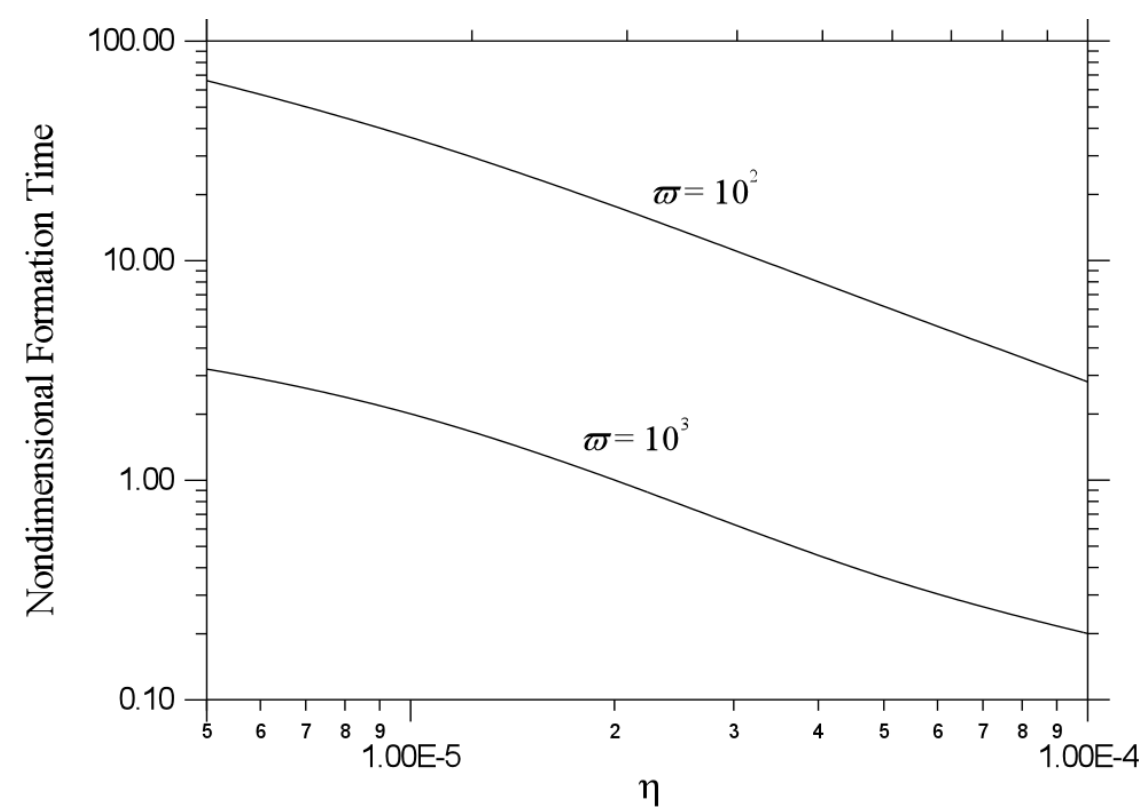

Figure 9: Formation time $t_{\mathrm{F}}$ versus particle inertia $\left(\gamma / \varpi=3 \times 10^{2}, \operatorname{Pr}=8, \operatorname{Ra}=0, \operatorname{Ra}_{\omega}=1.58 \times 10^{4}, \xi\right.$ $=1.85, \phi=0)$. [ $\mathrm{t}_{\mathrm{F}}$ defined as the time required for the particle structures to reach a constant size, a further increase in time simply causing a periodic displacement of such structures along the direction of imposed vibrations with no modification in terms of size].

Let us recall that for the considered phenomena vibrations will be exerting their influence on particles via two separate mechanisms, one being the "direct effect" of the resulting accelerations on the particles due to their different density with respect to the surrounding fluid and the other being related to the way by which oscillatory flow of thermovibrational nature tends to transport particles according to their size and drag.

The translation of the curve (Fig. 9) towards higher values of the time for decreasing $\varpi$ clearly indicates that thermovibrational effects also play a crucial role. When the shaking is applied, the transient leading to the final pattern and its duration depend strongly on both vibration frequency and particle size. As shown in Fig. 9, the formation time scales as $(\eta \varpi)^{-1}$.

The inertia parameter, however, has also a strong impact on the characteristic extension of the particle aggregates (which behaves as a decreasing function of $\eta$, see Figs. 10).

Under a slightly different perspective, these concepts could be further illustrated by highlighting that because the mass and size of particles have an influence on the "efficiency" by which the convective flow is able to transport them (or, in other words, on the ability with which particles are 
able to "follow" this oscillatory flow), they will definitely have an important role also in the processes which finally determines the extension of the accumulation regions. More precisely, because these inertial particles interact with the fluid through viscous Stokes drag and, therefore, the motion of particles at a given value of the Stokes number $\mathrm{St}_{2}$ typically lags behind that that would be typical of tracer particles moving with $\mathrm{St}_{1}<\mathrm{St}_{2}$, an increase in $\mathrm{St}$ (i.e. $\eta$ for a fixed density ratio) must be expected to decrease the extension of the final particle accumulations regions (Lappa, 2014b).

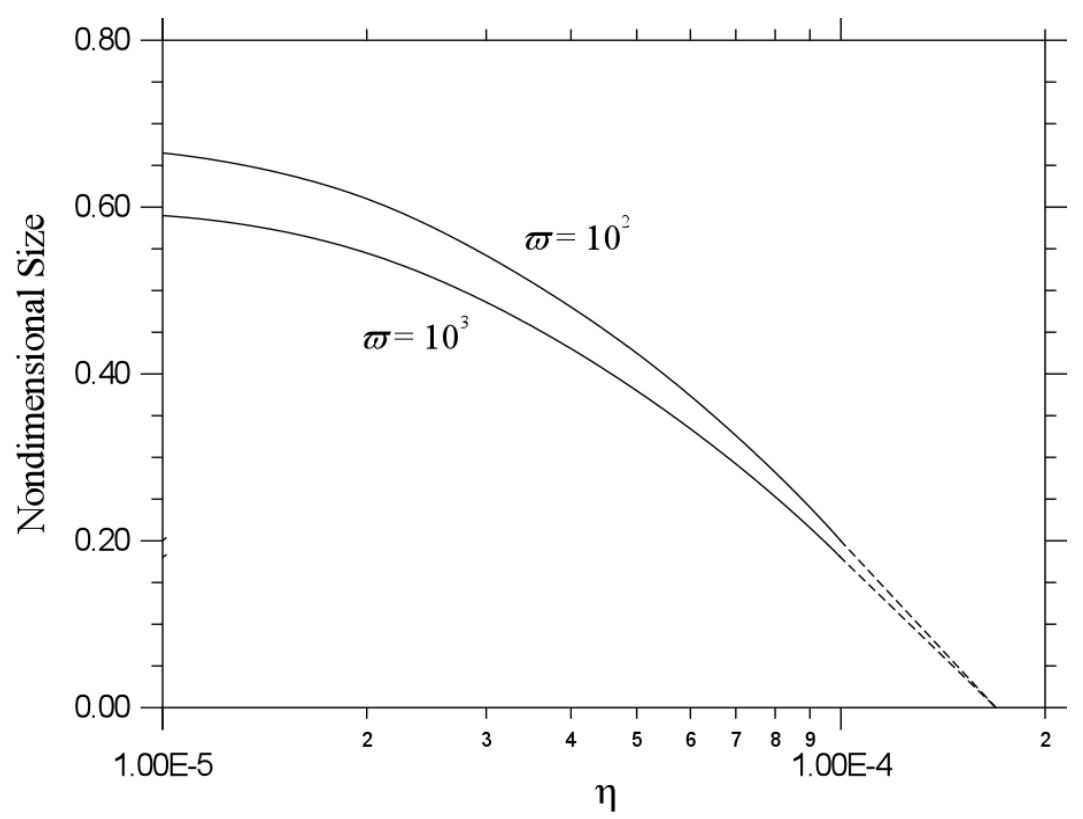

Figure 10: Characteristic size of particle aggregates versus inertia parameter $\left(\gamma / \varpi=3 \times 10^{2}, \operatorname{Pr}=8\right.$, $\left.\mathrm{Ra}=0, \mathrm{Ra}_{\omega}=1.58 \times 10^{4}, \xi=1.85, \phi=0\right)$. Linear extrapolation of the characteristic size to zero gives $\eta \cong 1.6 \times 10^{-4}$, which is in agreement with the absence of recognizable extended particle structures in numerical simulations performed for $\eta=2 \times 10^{-4}$.

The most remarkable outcome of all such arguments is that, because the underlying mechanism for particle accumulation does not require any kind of particle (hydrodynamic) interactions or attractive forces, the only necessary prerequisites being the existence of a temperature gradient and some inertial "disturbances" (a cyclic forcing) affecting the considered domain, these dynamics might play a role in the dilute medium represented by the mixture of gas and solid matter of primordial nebule and solar systems where, in principle, a variety of inertial disturbances should be operative at different length and time scales. As outlined in the preceding pages, such disturbances are known to be induced by a variety of effects, among them let us recall: a) tidal phenomena (as an example, Goodman, 1993, identified a tidally induced parametric instability in astrophysical disks due to the coupling between noncircular orbital excursions, which are pressure-modified epicycles, and inertial waves, which are also, in essence, pressure-modified epicycles), b) the differential character of Keplerian rotation (able to produce pulsations forces of various natures, Balbus and Hawley 1998) and c) the intrinsic degree of turbulence of the gas-dust disk (Gammie, 1999; Adams and Laughlin, 2000). 


\section{The role of Hydrodynamic and Electrostatic Interactions}

Having finished a sketch of particle self-assembly promoted by inertial effects in dilute systems (relatively small particle concentration), we turn now to consider the opposite situation in which particle-to-particle interplay is expected to be important. A synthetic description of the salient outcomes of such studies is reported in the remainder of this section together with a critical discussion of some accompanying necessary concepts provided to support the reader who may not be an expert in these fields. Obviously, we still give emphasis to results provided by microgravitybased research.

Indeed, a "parallel" branch of investigation also exists, yet dealing with "mechanically forced" systems, in which rather than being the mere outcome of spontaneous inertial effects (depending on the nature and properties of the background flow and on particle density and size), attractive or repulsive events and related patterning behaviour occur essentially as a result of the hydrodynamic interference among different particles. Such interactions, which require neither the existence of a "carrier" flow nor fluid/solid density difference, are mediated by the interstitial fluid and disappear in the limit as such a fluid is removed (i.e. particles in absolute vacuum).

Remarkably, such fluid-mediated interactions can be tuned to produce a fascinating variety of dynamical phenomena (as the presence of an independent background flow is not a requirement for such events to occur, in particular, we concentrate on the case of isothermal fluids).

The identification of the fundamental principles driving such phenomena dates back to the works of Tabakova and Zapruanov (1982) and Langbein (1991). Since then, several experimental and numerical studies have been carried out showing intricate scenarios of transition and complex regimes.

A seminal experimental work focused on these effects was published by Petit and Noetinger (1988), who clearly identified shear-induced particle "crystallization" phenomena driven by alternating shear stress in macroscopic suspensions (observations were reported on structures formed by glass spheres in silicon oils in normal gravity conditions). Tirumkudulu et al. (1999) continued this line of inquiry using suspensions of monodisperse neutrally buoyant spherical particles, thereby demonstrating that (solid versus liquid) density difference and gravity are not necessary ingredients. The suspension was indeed observed to separate itself into alternating regions of high and low particle concentration along the length of the domain. The field reached a certain level of maturity with the works of Wunenburger et al. (2002) and Zhu and Ma (2002), who reported on striped domain structures either parallel or perpendicular to the oscillatory direction depending on the considered oscillatory frequency $\omega$ and the amplitude $\gamma$ of the vibrational acceleration. Thomas and Gollub (2004) and Klotsa et al. (2007 and 2009) conceived and executed additional experiments that elegantly illustrated the principles responsible for such dynamics.

These problems are still a challenging task for numerical simulation, have instigated much research, and have led to many different solutions. The related mechanisms have been placed in a precise theoretical context over recent years (the reader being referred to the excellent studies by Thomson 
et al., 1997; Ivanova and Kozlov, 2002; Kozlov et al., 2006; Hassan and Kawaji, 2008; Saadatmand and Kawaji, 2010).

As an example, Thomson et al., (1997) studied theoretically the motion induced on particles that are suspended in an incompressible fluid by an externally imposed random acceleration field (they assumed a process obeying Gaussian statistics, consistent with the assumption that many independent sources contribute to the acceleration field typical of a space platform such as the ISS). These authors demonstrated the existence of a hydrodynamically induced attraction between pairs of particles at distances large compared with their radii, and repulsion at short distances.

Kozlov et al. (2006) provided some additional knowledge on these effects by showing that the hydrodynamic interaction affecting close particles is driven by a vibration-induced viscous force, which in some circumstances results in an attraction between the particles in the direction of vibrations and a repulsion between the particles in the direction perpendicular to the vibrations direction. This effect can lead, in a very concentrated suspension, to a compression of particles in the direction of vibrations and in their extension in the direction perpendicular to the vibration direction.

It is worth noting that in case of a nonviscous fluid the mean hydrodynamic interaction of particles in the oscillating flow has the opposite sign with respect to the phenomena described above (Lamb, 1932). The change of interaction and attraction of particles in the vibration direction takes place only in viscous liquids at a short distance (as also shown by Tabakova and Zapruanov, 1982). This implies that the above mechanism works only if the condition of "viscous hydrodynamic interaction" between particles is ensured during oscillations, which reads $\omega \ell^{2} / v<1$. It means that the distance $\ell$ between the particles has to be shorter than the distance $\delta=\sqrt{v / \omega} \propto R / \sqrt{S t_{v}}$. At larger $\ell$, the hydrodynamic mutual interference is negligible or it is replaced by the opposite effect (Ivanova et al., 2005). This is in agreement with the typical results of space experiments (see, e.g., Sun et al., 1994 and Ellison, 1995) where the effects of high-frequency g-jitters have generally been observed to be rather "localized".

As the reader might expect at this stage, the major outcome of such theoretical efforts has been the derivation of precise mathematical expressions relating the hydrodynamic forces induced by vibrations to the physical properties of the considered solid-fluid system over a wide spectrum of conditions (viscous or inviscid fluids, low or high frequencies, dilute or concentrated suspensions, etc.). Most recently there has been an upsurge of interest in all these dynamics owing to the discovery of new patterns and intriguing spatiotemporal phenomena.

For instance, Voth et al. (2002) reported additional interesting findings on the fascinating mechanisms that may promote the self-assembly of ordered structures in a vibrated isothermal liquid. These authors conducted experiments using a rigid container vibrated vertically by an electromagnetic vibrator containing a viscous fluid and a sub monolayer of uniform stainless steel spheres. The resulting patterns included hexagonally ordered micro-crystallites, time-periodic structures, and chaotic fluctuating patterns with complex dynamics (Fig 11) according to the considered value of the non-dimensional acceleration amplitude $\gamma=b \omega^{2} / g$. 
For three interacting particles the system was found to form an asymmetric pattern with two paired and one distant particle as shown in Fig. 11(a). In a configuration with seven particles, stable hexagonal states formed over the range $2.8 \leq \gamma \leq 3.0$ (Fig. 11b). At slightly higher acceleration, a time dependent state was observed with two central particles rotating inside a ring of five others (Fig. 11c). For larger numbers of particles, the presence of both attraction and repulsion led to even more complex many-body effects, which were generally time dependent. As shown in Fig. 11(d) $(\gamma=3.7)$, particles near the centre of the cluster remain in contact even when particles near the periphery separate. At $\gamma=3.9$, (Fig. 11e) the inner particles have separated and the system forms a bound state with weakly chaotic motion of all the particles, a mesoscopic liquid.

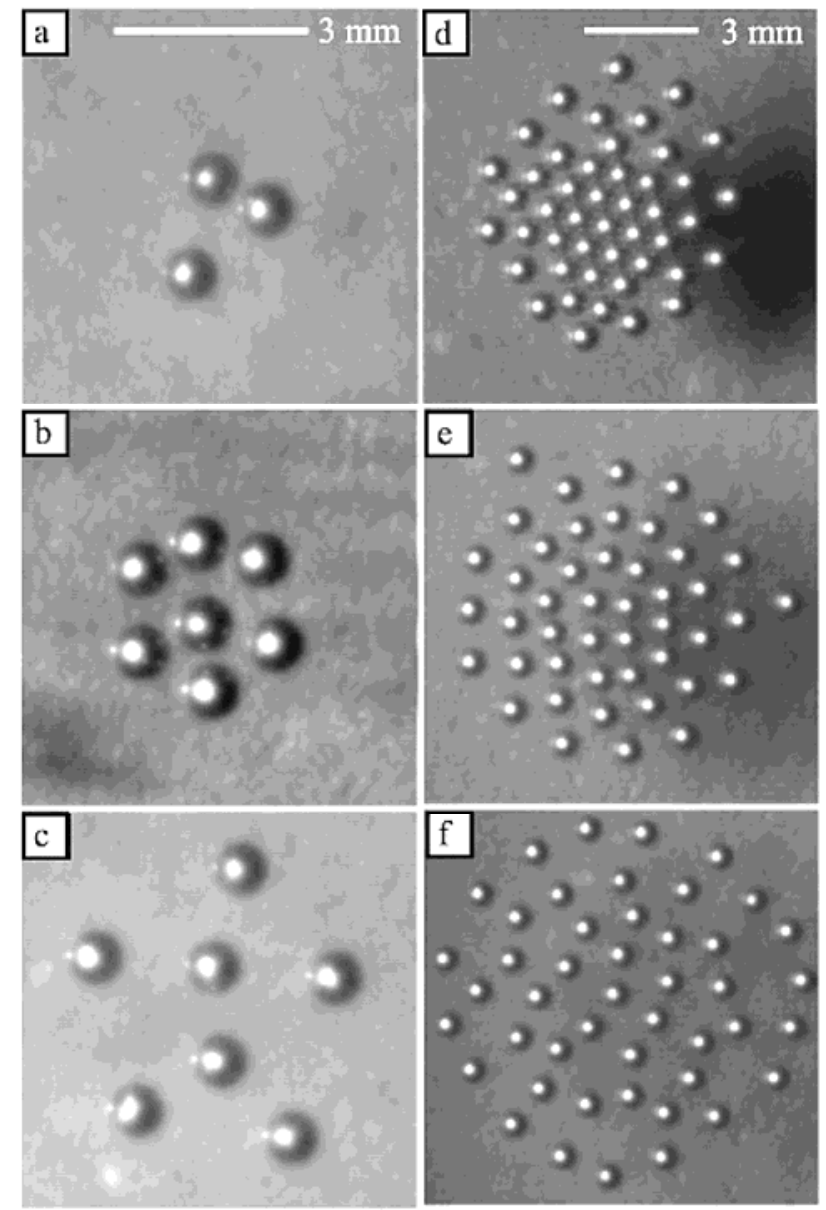

Figure 11: Patterns formed by multiple particle systems when both attractive and repulsive hydrodynamic interactions are important (water/glycerol mixture of kinematic viscosity 8 [cSt] and a sub monolayer of uniform stainless steel spheres of radius $\mathrm{R} \cong 4$ [mm], density ratio $\xi \cong 7$, different values of $\gamma=b \omega^{2} / g$ ): (a) Three particles at $\gamma=3.0$; (b) Seven particles in a stable hexagon at $\gamma=3.0$; (c) Seven particles in stable time-periodic motion at $\gamma=3.7$; (d) Many particles at $\gamma=3.7$, where the central particles are in contact and exterior particles are held apart by the hydrodynamic repulsion; (e) At $\gamma=3.9$ all the particles have separated and form a bound liquid; (f) $\gamma=5.3$, apparently chaotic behaviour. Reprinted figure with permission from Voth G. A., Bigger B., Buckley M.R., Losert W., Brenner M.P., Stone H.A., and Gollub J.P., Phys. Rev. Lett., 88, 234301 (2002), Copyright (2002) by the American Physical Society, http://dx.doi.org/10.1103/PhysRevLett.88.234301. 
As a further more recent example, by investigating experimentally the behaviour of two identical spheres suspended magnetically in a fluid to mimic weightless conditions, Pacheco-Martinez et al. (2013) observed the spheres to spontaneously orbit each other under the influence of mutual attraction for sufficiently large vibration amplitudes.

Although the problem is very complex and far to be well understood, it might even be the case that similar effects were at work in the early stages of planetesimal formation, provided the interstitial gas was sufficiently dense to promote such a hydrodynamic interplay.

The most remarkable discovery in microgravity with relevance to the mechanisms driving planet formation in the centimeter-sized grain range, however, is due to Donald Pettit, a mission specialist onboard the ISS Expedition 6 in 2002 and 2003 (see Love and Pettit, 2004; Tytell, 2004; for other microgravity experiments dealing with $1[\mu \mathrm{m}]$ particles for which Brownian dynamics in the free molecular flow regime are expected to be important, the reader is referred to the earlier excellent study by Blum et al., 2000). During his six-month stay aboard the space station, this astronaut conducted some qualitative experiments showing how fluids react in an extremely low gravity environment. These experiments revealed that particles of various materials, which varied in size up to $6[\mathrm{~mm}]$, could naturally clump together in microgravity when confined to a volume that included a few grams of the materials.

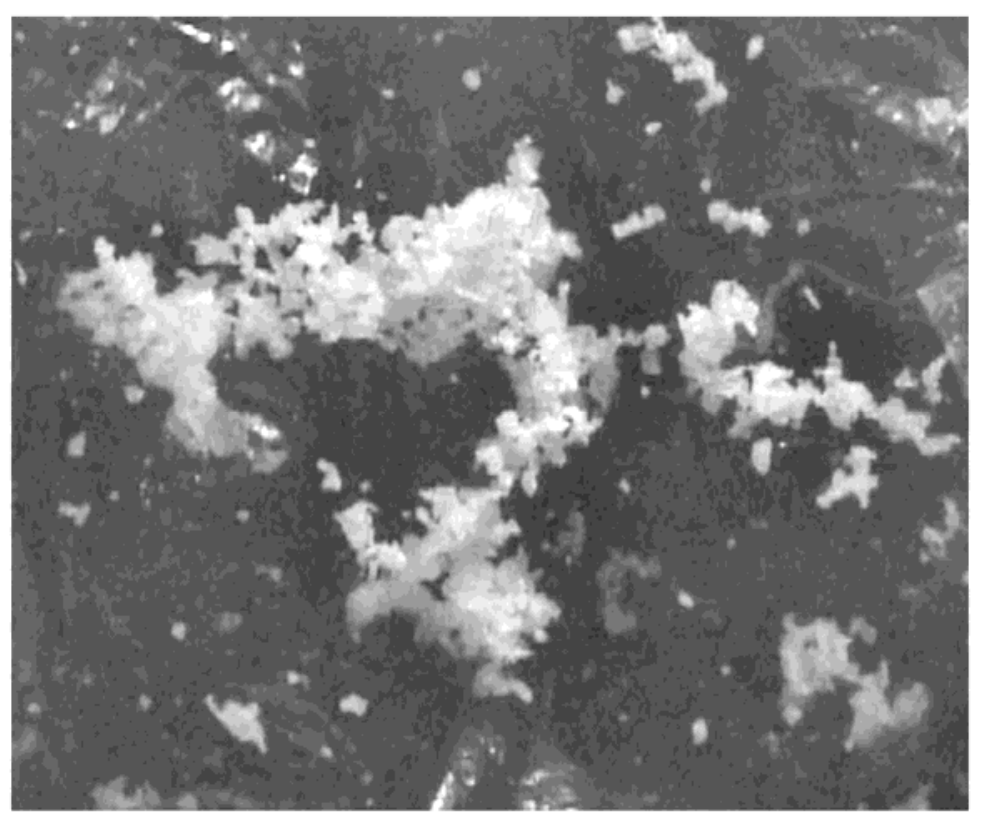

Figure 12: Aggregates of 1- to 6-mm salt $(\mathrm{NaCl})$ particles in air (approximately 1:1 scale) formed in microgravity conditions (Courtesy of D.R. Pettit, Lyndon B. Johnson Space Center, NASA).

The granular solids used for these tests were limited to those readily available aboard the ISS, namely: table salt $(\mathrm{NaCl})$ crystals $0.5-1.0[\mathrm{~mm}]$ in size, $\mathrm{NaCl}$ crystals $\cong 1-6[\mathrm{~mm}]$ in size, sugar crystals $\cong 1[\mathrm{~mm}]$ in size, powdered coffee particles $\cong 0.1[\mathrm{~mm}]$ in size, and mica flakes $\cong 5[\mu \mathrm{m}]$ in diameter. 
Although these specific materials are obviously not important in planet formation, they cover a relatively large range of materials type, including a crystalline ionic solid, two different organic substances, and a hydrated silicate.

A sample of each material, with a mass of a few grams, was placed in an inflated plastic bag of $\cong 4$ [litres] volume (the classical ziplock bag used in space by astronauts to collect or store items of various natures and sizes). Once sealed, the bag was shaken strongly to separate the particles and destroy any pre-existing structures. Although most of these experiments were qualitative, they provided a convincing proof that the mm-sized solid particles could aggregate into $1-5$ [cm] fractallike clusters within a few seconds (Fig. 12). The aggregates broke up into individual particles when shaken vigorously, but new clusters were seen to form quickly when the shaking was stopped. The material properties of the solids appeared to have a scarce effect on the observed behaviour.

The spontaneity, speed, and repeatability of the process suggests that it was strongly supported by the existence of inter-particle forces (identifiable as electrostatic in nature given the very long times generally required for vibration-induced hydrodynamic interactions to produce particle selfassembly).

It was realized that the container did affect the experiment by keeping particles confined at a relatively high density ( $\cong 10^{3}\left[\mathrm{~g} \mathrm{~m}^{-3}\right]$ ), thus increasing collision frequency and promoting such electrostatic (contact) interactions.

Some experiments were based on mica flakes suspended in water rather than air. Interestingly, in this case, the much greater density and viscosity of the fluid led to the same general clustering behaviour, but over a period of days rather than seconds.

Albeit qualitative in nature, these experiments revealed the counterintuitive sticking behaviour of solid particles in gas without the influence of gravity.

To do justice to these interesting findings (what sets this class of phenomena apart from the others considered in this work is the essentially electrostatic nature of the involved forces), the remainder of this section is devoted entirely to get quantitative details and additional insights into the related mechanisms. Towards this end, we discuss the preparation (together with some related physical reasoning) and execution of some novel (still unpublished) experiments expressly conceived to reproduce such behaviours in normal gravity conditions.

Because experiments with particles in air are rather difficult on the ground due to the large density difference between the fluid and the dispersed phase, we based such attempts on water (as working fluid) and neutrally buoyant tracers.

Particles (carboxylate solid spheres having diameter $1 \mu \mathrm{m}$ made of latex) were initially dispersed in water contained in a cylindrical glass phial (internal diameter $20[\mathrm{~mm}]$, filled up to a height of liquid $20[\mathrm{~mm}]$ from the bottom).

Instead of shaking the suspension (as in the experiments onboard the ISS), we stirred the mixture of liquid and dispersed particles with a stirring velocity of 1 [rps] applied for approximately 4 or 5 seconds in order to "mimic" conceptually vorticity present in typical industrial processes such as those discussed in the introduction or in contexts with astrophysical relevance. 
In agreement with the earlier microgravity findings, we could observe cluster formation neither in the first few seconds (after the beginning of the experiment), nor in the following minutes. Unfortunately, longer times of observation were not allowed by the density difference (although very small) between the liquid and the tracers (by which particles were slowly removed from the liquid when agitation was discontinued and transported towards the top of the container).

Perhaps, the most convenient way to start a discussion to fully understand the mechanisms that lead to this (negative) occurrence (in order to use them advantageously for the ensuing design of a successful experiments) is to observe that the amount of charge generated during the handling and processing of a liquid depends on several factors.

Such factors include the flow characteristics of the liquid, i.e. flow velocity, stirring, shear and turbulence, and the mobility of liquid ions.

More precisely, while contact electrification is believed to be the manner in which contacting solid surfaces become electrostatically charged and represents a useful general model of electrostatic charging, the actual mechanism by which fluids (more precisely, liquids) become electrostatically charged is known as double-layer charging (Glor and Luttgens, 1989). This can occur at liquid solid and liquid-liquid interfaces, such as where a liquid makes contact with the wall of a pipe or vessel, or with suspended particles. This effect, combined with the competing effect of ion recombination at the pipe or vessel wall can result in an unbalancing of charge within the liquid if it is insulating in electrostatic terms or sufficiently isolated from electrical ground by vessels that are made from an electrically insulating material, such as plastic (Kozman and Gavis, 1962).

The principal indicator of ion mobility in liquids is the electrical conductivity, which is typically reported in units of Siemens per meter $\mathrm{m}\left[\mathrm{Sm}^{-1}\right]$ or PicoSiemens per meter $\left[\mathrm{pSm}^{-1}\right]$. Although glass is a relatively good (although not perfect) insulator from an electrostatic-chargedispersion perspective, water has a relatively high electric conductivity $\left(1 \times 10^{9}\left[\mathrm{pSm}^{-1}\right]\right.$ at $\left.25^{\circ} \mathrm{C}\right)$, which makes the $\mathrm{Q}$ parameter defined by eq. (13) relatively small (of the order $\mathrm{O}\left(10^{-8}\right)$ for the conditions considered in our experiments).

Because, as explained in Sect. 2, the nondimensional parameter $Q$ should be regarded as a "measure" of the ability of the considered flow to "retain" electric charges eventually produced in the fluid by large-scale flow and related "frictional" effects (before they are transferred to ground through the non-perfectly insulating walls of the container), the related small value attained by this parameter should be regarded as a possible justification for the absence of particles aggregates in our experiments (at least in the reduced timeframe in which we observed the dynamics).

In order to test the validity of these arguments we repeated the experiments mitigating the too high conductivity of water by the addition of a miscible liquid characterized by a much smaller value of the electric conductivity (along these lines, let us recall that the microgravity experiments were conducted in air, that at ordinary temperatures behaves as an excellent electrical insulator). In particular, we selected ethyl alcohol, its electric conductivity being four orders of magnitude smaller than that of water $\left(1.35 \times 10^{5}\left[\mathrm{pSm}^{-1}\right]\right.$ at $\left.25^{\circ} \mathrm{C}\right)$. 
For a percentage of ethyl alcohol around 30\% we could immediately observe the formation and apparently endless growth of "fractal aggregates" (often in the form of long irregular chains, formed in the bulk of liquid and then transported towards the glass wall of the phial by the residual centrifugal force (due to the initial "stirring" applied to the liquid to promote electrostatic interactions) and the electrostatic effect itself, where they could "attach" one another giving rise to a sort of porous "lattice", Fig. 13).

Although it is obvious that further elaboration of such models will be required in the future, in particular to account for the effects of friction on gases, this being necessary for an accurate quantitative description of the experiments originally executed on the ISS, it is worth highlighting that our ground experiments successfully reproduced most, although not all, features of the tests carried out with particle in air in orbit.

In this context it is also worth mentioning the parallel line of inquiry originating from the experiments of Manley et al. (2004), Zhu et al. (2005) and Castellanos (2005), who further addressed the role of van der Waals forces in producing aggregates in terrestrial experiments with solid particles. These studies showed that cohesive particles can be agglomerated in a fluidized state due to the strong interparticle attractive forces as compared to particle weight. In the experiments by Castellanos (2005), in particular, the drag force created by an upwards flowing gas was expressly used to counteract gravity and investigate how particles with size of the order $\mathrm{O}(10)[\mu \mathrm{m}]$ can form fractal structures. In these systems, fractal aggregates size was limited by the fact that gravity is a volume force whereas drag acts on the surface. Thus, a size limit was found for aggregate size depending on the ratio of interparticle van der Waals force to particle weight (the relative importance of these effects being measured by the so-called granular Bond number). The comparison of results obtained in microgravity and on Earth definitely demonstrated that cluster growth is limited by gravity-induced restructuring (Manley et al., 2004).

Subsequent works (Castellanos et al., 2005 and Valverde and Castellanos, 2006) have been devoted to formulate a generalized relationship between the particle volume fraction, the interparticle attractive force and the aforementioned granular Bond number.

These fine powders have been the subject of active investigation in the recent years due to their novel physical, chemical and mechanical properties. Their uniqueness arises from their high ratio of surface area to volume, providing high fluid-solid contact and reaction efficiencies but, on the other hand, enhancing the effect of interparticle attractive forces that inhibit powder flow. When the size of the constituent particles decreases below a critical value (typically $\cong 50 \mu \mathrm{m}$ ) interparticle attractive forces become larger than particle weight, leading to the formation of stable arches that hamper flowability and give rise to highly porous structures similar to those shown in Fig. 13c. 

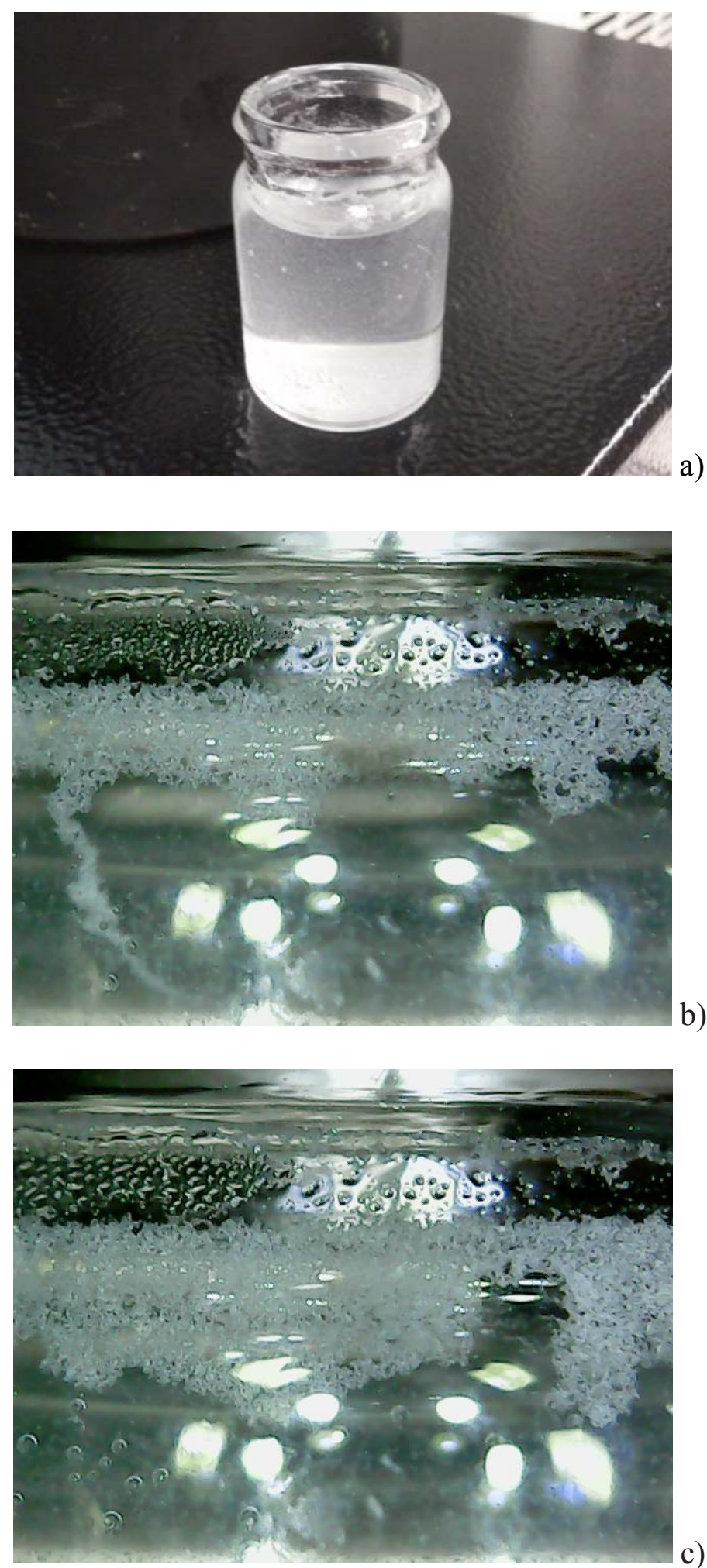

Figure 13: Simulation of microgravity experiments in normal gravity conditions: a) neutrally buoyant particles dispersed in a solution of water and ethanol contained in a glass phial after the application of 5 [s] stirring at 1 [rps] (some larger-size aggregates can be distinguished in the bulk); b) detail of particle aggregates at the liquid-glass lateral boundary after 1 minute (a chain of particles extended from the glass surface to the interior of the liquid can be distinguished on the left side of the picture); c) after 5 minutes (due the continuous addition of fractal aggregates formed in the bulk, a kind of porous "lattice" is clearly visible at the wall). 


\section{Discussion and Conclusions}

There is no doubt that at the present stage the results obtained in microgravity are mostly of fundamental nature, quantifying theoretical models of gravity influences on fluid phenomena through comparison with terrestrial experiments or leading to better insights into the significance of forces and interactions which, during experiments on Earth are masked by gravity.

In particular, by removing the influence of gravity, scientists are more closely observing still poorly known influential processes that are thought to be responsible for structure formation during the processing of solid-fluid mixtures.

In such a context, the recent identification of "new" families of particle attractors, together with the related intrinsic "multiplicity" and sensitivity to particle inertia is of enormous conceptual significance as it opens new fascinating (heretofore unexplored) perspectives in the investigation of a variety of subjects, ranging, just to cite some examples, from mechanisms operating at very large scales (e.g., related to the initial stages of planet formation driven by the accumulation of small dust particles), to all small-scale technological applications which simply share the use of microfluidic devices to manipulate the transport of tiny particles.

It is obvious that the study of pattern formation driven by such mechanisms also falls under the broader heading of nonequilibrium phenomena. Beyond practical applications, it is therefore clear that these problems also exert an appeal to researchers and scientists as a consequence of the complexity of the possible stages of evolution, of the nonlinear behaviour and because these organized structures are aesthetically and philosophically pleasing as well as irresistible to theoretical physicists.

Because, obtaining a robust understanding of reality requires a diversity of model types, ranging from simple to complex, in which various processes can be turned on and off and the results carefully diagnosed (this is called a modeling hierarchy and its use forms the backbone of forward progress in any field), we have considered four distinct categories of particle accumulation structures supported by microgravity conditions, exploring separately a vast range of physical effects and related characteristic parameters.

Starting from theoretical models able to explain how inertial particles initially dispersed in a fluid organize themselves into what appear to be travelling threads or filaments (1D objects) or discrete stationary collections of sharp stripes (2D accumulation planes) under the influence of isolated waves or interacting (counter-propagating) waves, respectively, then we have moved to a different class of inertial phenomena in which the presence of waves as the main particle-accumulation driving mechanism is replaced by "vibrational" convective flows.

While in the first situation, particle preferential segregation can be explained in terms of a "phase locking" process between the travelling fluid-dynamic disturbance and the typical frequency of motion or rotation of a generic particle (as a result of which, the motion of an individual particle in the $3 \mathrm{D}$ flow gets synchronized with the wave), in the latter case particle self-assembly is due to the 
delicate interplay between particle inertia and oscillatory thermovibrational effects, which tends to constrain particles to specific regions of the physical space.

The subsets of space where such phenomena occur (the so-called "attractors" using a terminology borrowed from the general field concerned with the study of non-linear systems) evolve accordingly from the 1D dimensional paths or planar areas seen in the case of waves, to geometric objects which seem to resemble the typical "quadrics" of projective geometry. Indeed, by varying the relative direction of imposed vibrations the shape of the accumulation regions changes from cylindrical surfaces to a compact surfaces passing through the intermediate stage of a conical surface. Just as in geometry the ellipsoid and the elliptic paraboloid, are non-degenerate compact quadric surfaces, while the cone and the cylinder are equivalent to the so-called degenerate form, the particle surfaces revealed by these studies might be the signature of some hidden analytic order (algebraic equation) underlying the formation and existence of such attractors (which would deserve additional attention).

Whenever possible, we have used fluid vorticity as a unifying (cardinal) concept for the interpretation of the considered dynamics. Although the role of this quantity in determining inertial particle association phenomena (in conjunction with more or less pronounced levels of turbulence) has been already recognized in the past, the new knowledge provided by microgravity research seems to indicate that, by limiting to the models traditionally used in the literature, one may be omitting important components of the general dynamics of realistic systems (in nature or technology, where a variety of fluid-dynamic and inertial (acceleration) disturbances might be present at the same time).

To some extent the examples examined here may help the reader in abstracting from specific cases features which are essential in the description of the general phenomenon of particle self-assembly in other circumstances (not necessarily related to these examples), thereby allowing most of the concepts we have illustrated to spread from their initial heartland of microgravity flows and related materials-science problems to a more general (perhaps universal) context.

This is especially true for problems related to the formation of planetary systems, which are intimately tied to the question of the evolution of the gas and solid material in the early nebula. Although the dynamical behaviour of micrometer-sized particles is expected to be dominated by the gas drag, while meter-sized bodies are allowed to decouple from the gas and move on Keplerian orbits, it is known that a transition regime of particle sizes exists where the influence of the gas drag, gravity, and the inertia forces of the particles are on same order of magnitude.

Still in the case of solid-liquid systems subjected to the perturbing action of inertial disturbances (in the form of vibrations), we have also provided some effort to discuss the influence of particle interactions. It is expected that interparticle attractive or repulsive behaviours driven by local forces of hydrodynamic nature (mediated by the interstitial fluid in concentrated suspensions) will also play a significant role in determining the distribution of dispersed solid matter (inducing global compression or expansion of the resulting pattern and/or leading to local phenomena such as the emergence of network-like regions and ordered micro-crystallites, particles orbiting each other or 
particles bound together with weakly chaotic motion), thereby contributing to the complexity of the resulting dynamics.

As another physical connection in which frictional effects induced by stirring (vorticity) or vibrations (shaking) might be also important, we have considered circumstances where particle adhesion is promoted by electrostatic interaction (supported by a charging process of "frictional nature").

The resulting structures (emerging in the form of fractal aggregates) display notable affinity with those generally provided by numerical studies of inertial particles suspended in isotropic and homogeneous turbulent flows (see, e.g., Bec, 2005 and Bec et al., 2007). The fact that similar structures are observed across two completely different categories of particle phenomena (the first being driven by inertia, the second by electrostatic effects) may be considered as the typical signature of the strongly dissipative nature of the process driving accumulation for both cases (when the geometry of inertial particle clusters in developed turbulence is controlled by the dissipative effective dynamics of the particle motion at small scale, attractors are generally fractals).

Since many readers of this journal may not have previously encountered the phenomena described here, our main goal was to stake out some common ground by providing a synthetic review of the underlying mechanisms and cause-and-effect relationships, under the optimistic idea that the contacts established in the present survey between the fields of microgravity research (and associated materials-science problems) and astrophysical and geophysical fluid-dynamics will develop into an on-going, mutually beneficial dialogue. Along these lines, we have cited so many publications expressly to stimulate further exchange and cross-fertilization of knowledge by encouraging readers to follow up various details that could not be treated in depth here due to page limits.

\section{Acknowledgement}

We would like to thank P. Capobianchi for the kind support provided in executing the experiments shown in Figure 13.

\section{References}

[-] Abshagen J., Lopez J. M., Marques F. and Pfister G., (2005), Mode competition of rotating waves in reflectionsymmetric Taylor-Couette flow, J. Fluid Mech., 540, 269-299.

[-] Abshagen J., Lopez J. M., Marques F. and Pfister G., (2008), Bursting dynamics due to a homoclinic cascade in Taylor-Couette flow, J. Fluid Mech., 613, 357-384

[-] Adams F. C. and Watkins R., (1995), Vortices in circumstellar disks, The Astrophysical Journal, 451, $314-327$.

[-] Adams F. C., and Laughlin G., (2000), Protostellar Disk Formation and Early Evolution, Space Science Reviews, 92, 23-38.

[-] Ayala O., Rosa B., Wang L-P., (2008), Effects of turbulence on the geometric collision rate of sedimenting droplets. Part 2. Theory and parameterization, New J. Phys., 10, 075016.

[-] Babiano A., Cartwright J. H. E., Piro O. and Provenzale A., (2000), Dynamics of a small neutrally buoyant sphere in a fluid and targeting in Hamiltonian systems, Phys. Rev. Lett., 84, 5764-5767.

[-] Balbus S.A. and Hawley J.F., (1998), Instability, turbulence, and enhanced transport in accretion disks, Reviews of Modern Physics, 70(1), 1-53.

[-] Balkovsky E., Falkovich G., and Fouxon A., (2001), Intermittent Distribution of Inertial Particles in Turbulent Flows, Phys. Rev. Lett. 86, 2790-2793. 
[-] Balmforth N. J. and Spiegel E. A., (1995), Sinuous Modes and Steady WARPS of Polytropic Disks, Waves in Astrophysics, 773, 55-69

[-] Balvin M., Sohn E., Iracki T., Drazer G., and Frechette J., (2009), Directional locking and the role of irreversible interactions in deterministic hydrodynamics separations in microfluidic devices, Phys. Rev. Lett. 103, 078301.

[-] Barge P. and Sommeria J., (1995), Did planet formation begin inside persistent gaseous vortices?, Astronomy and Astrophysics,. 295(1), L1-L4

[-] Barranco J. A. and Marcus P. S., (2000), Vortices in protoplanetary disks and the formation of planetesimals, Center for Turbulence Research-Proceedings of the 2000 Summer Program (2000), 97-108.

[-] Bec J., (2005), Multifractal concentrations of inertial particles in smooth random flows, J. Fluid Mech., 528, $255-277$.

[-] Bec J., Biferale L., Cencini M., Lanotte A., Musacchio S., and Toschi F., (2007), Heavy Particle Concentration in Turbulence at Dissipative and Inertial Scales, Phys. Rev. Lett., 98, 084502.

[-] Benczik I. J., Toroczkai Z., and Tél T., (2002), Selective Sensitivity of Open Chaotic Flows on Inertial Tracer Advection:Catching Particles with a Stick, Phys. Rev. Lett., 89 (16), 164501

[-] Biferale L., Moffetta G., Celani A., Lanotte A., Toschi F., (2005), Particle trapping in three-dimensional fully developed turbulence, Phys. Fluids, 17, 021701 (2005), 4 pages

[-] Blum J., Wurm G., Kempf S., Poppe T., Klahr H., Kozasa T., Rott M., Henning T., Dorschner J., Schräpler R., Keller H. U., Markiewicz W. J., Mann I., Gustafson B. A., Giovane F., Neuhaus D., Fechtig H., Grün E., Feuerbacher B., Kochan H., Ratke L., El Goresy A., Morfill G., Weidenschilling S. J., Schwehm G., Metzler K., and Ip W.-H. (2000). Growth and Form of Planetary Seedlings: Results from a Microgravity Aggregation Experiment, Phys. Rev. Lett., 85,2426-2429.

[-] Boffetta G. and De Lillo F., (2004), Large scale inhomogeneity of inertial particles in turbulent flows, Phys. Fluids, 16(4), L20-L23

[-] Bracco A., Chavanis P. H., Provenzale A., Spiegel E. A., (1999), Particle aggregation in a turbulent Keplerian flow, Phys. Fluids, 11( 8), 2280-2287

[-] Cabot W. and Pollack J. B., (1992), Direct simulations of turbulent convection. II. Variable gravity and differential rotation, Geophys. Astrophys. Fluid Dynam., 55, 97-133.

[-] Cameron A. G. W., (1978), Physics of the primitive solar accretion disk, Moon and the Planets, 18, 5-40.

[-] Carotenuto L., Piccolo C., Castagnolo D., Lappa M., Garcìa-Ruiz J.M., (2002), Experimental observations and numerical modelling of diffusion-driven crystallisation processes, Acta Crystallographica D, 58, 1628-1632.

[-] Castellanos A., (2005), The relationship between attractive interparticle forces and bulk behaviour in dry and uncharged fine powders, Advances in Physics, 54(4), 263-376.

[-] Castellanos A., Valverde J. M., and Quintanilla M. A. S., (2005), Physics of Compaction of Fine Cohesive Particles, Phys. Rev. Lett., 94, 075501 (4 pages).

[-] Chen L., Goto S. and Vassilicos J. C., (2006), Turbulent clustering of stagnation points and inertial particles, J. Fluid Mech., 553, 143-154.

[-] Clever R. M. and Busse F. H., (1981), Low Prandtl number convection in a layer heated from below, J. Fluid Mech., $102,61-74$.

[-] Cröll A., Kaiser Th., Schweizer M., Danilewsky A.N., Lauer S., Tegetmeier A., Benz K.W., (1998), Floating-zone and floating-solution-zone growth of GaSb under microgravity, J. Cryst. Growth, 191, 365-376.

[-] Cuzzi J. N., Dobrovolskis A. R. and Champney J. M., (1993), Particle-gas dynamics in the midplane of a protoplanetary nebula, Icarus, 106, 102-134.

[-] Deng X. and Chawla N., (2006), Modeling the effect of particle clustering on the mechanical behaviour of SiC particle reinforced Al matrix composites, Journal of Materials Science, 41(17), 5731-5734.

[-] Devenish B. J., Bartello P., Brenguier J-L, Collins L. R., Grabowski W. W., Ijzermans R. H. A., Malinowski S. P., Reeks M. W., Vassilicos J. C., Wang L-P., and Warhaft Z., (2012), Droplet growth in warm turbulent clouds, Quarterly Journal of the Royal Meteorological Society, 138 (667), 1401-1429.

[-] Dobrovolskis A. R., Cuzzi J. N. and Hogan R. C., (1993), Particle sorting and segregation in a preplanetary nebula, Bull. Am. Astron. Soc., 25, 1122.

[-] Eaton J.K. and Fessler J.R., (1994), Preferential concentration of particles by turbulence, Int. J. Multiphase Flow, 20, 169-209.

[-] Ellison J., Ahmadi G., Regel L. and Wilcox W., (1995), Particle motion in a liquid under g-jitter excitation, Microgravity Sci. and Tech., 8, 140- 147.

[-] Fein J.S. and Pfeffer R.L., (1976), An experimental study of the effects of Prandtl number on thermal convection in a rotating, differentially heated cylindrical annulus of fluid, J. Fluid Mech, 75, 81-112.

[-] Gammie C., (1999), Accretion Disk Turbulence, in Interstellar Turbulence, Vol. 1, p. 277, Cambridge University Press.

[-] Garnier N., Chiffaudel A. and Daviaud F. (2003a) Nonlinear dynamics of waves and modulated waves in 1D thermocapillary flows. I. General presentation and periodic solutions, Physica D, 174 (1-4), 1-29- II. Convective/absolute transitions, Physica D, 174 (1-4), 30-55

[-] Glor, M. and G. Luttgens, (1989), Understanding and Controlling Static Electricity, Expert- Verlag, Ehningen bei Boblingen, Federal Republic of Germany FR, p. 50. 
[-] Godon P. and Livio M.,, (1999), Vortices in protoplanetary disks, The Astrophysical Journal, 523,350-356.

[-] Goodman J., (1993), A local instability of tidally distorted accretion disks, Astrophysical Journal, Part 1,406(2), 596-613.

[-] Han B., Akeprathumchai S., Wickramasinghe S. R., Qian X. (2003). Flocculation of biological cells: Experiment vs. theory. AIChE Journal 49 (7), 1687-1701

[-] Hassan S. and Kawaji M., (2008), The Effects of Vibrations on Particle Motion in a Viscous Fluid Cell, J. Appl. Mech., 75, 031012 [7 pages].

[-] Haszpra T. and Tél T. (2011), Volcanic ash in the free atmosphere: A dynamical systems approach, Journal of Physics: Conference Series 333012008 (12 pages)

[-] Herrmann J., Karweit M., and Drazer G., (2009), Separation of suspended particles in microfluidic systems by directional-locking in periodic fields, Phys. Rev. E 79, 061404.

[-] Hiddessen A.L., Weitz D.A., and Hammer D.A., (2004), Rheology of binary colloidal structures assembled via specific biological cross-linking, Langmuir, 20(16), 6788-6795.

[-] Hide, R. and Mason, P. J., (1975), Sloping convection in a rotating fluid. Adv. in Phys. 24, 47-99.

[-] Hoyas S., Herrero H. and Mancho A. M., (2004), Thermocapillary and thermogravitatory waves in a convection problem, Theor. Comput. Fluid Dyn., 18 (2-4), 309-321.

[-] Huang L. R., Cox E. C., Austin R. H., and Sturm J. C., (2004), Continuous particle separation through deterministic lateral displacement, Science, 304(5673), 987-990.

[-] Ivanov I.B. and Kralchevsky P.A., (1997), Stability of Emulsions under Equilibrium and Dynamics conditions, Colloids \& Surfaces A, 128, 155-175.

[-] Ivanova A.A. and Kozlov V.G., (2002), Sand-fluid interface under action of vibration. Fluid Dynamics, 37 (2), $277-$ 293.

[-] Ivanova A.A., Kozlov V.G. and Kuzaev A.F., (2005), Vibrational lift force acting on a body in a fluid near a solid surface. Dokladi RAN, vol. 402 (4), pp. 1-4 (Translated: Doklady Physics, vol. 50 (6), pp. 311-314),

[-] Jing C.J., Tsukada T., Hozawa M. et al. (2004), Numerical studies of wave pattern in an oxide melt in the Czochralski crystal growth, J. Cryst. Growth, 265 (3-4), 505-517.

[-] Kley W., Papaloizou J. C. B., and Lin D. N. C., (1993), On the momentum transport associated with convective eddies in accretion disks, Astrophys. J., 416, 679-689.

[-] Klimpel R.C. and Hogg R., (1986),Effects of flocculation conditions on agglomerate structure, Journal of Colloid and Interface Science, 113(1), 121-131

[-] Klotsa D., Swift M. R., Bowley R. M., and King P. J., (2007), Interaction of spheres in oscillatory fluid flows, Phys. Rev. E 76, 056314

[-] Klotsa D., Swift M. R., Bowley R. M., and King P. J., (2009), Chain formation of spheres in oscillatory fluid flows, Phys. Rev. E 79, 021302

[-] Kokubo E. and Ida S., (2000), Formation of Protoplanets from Planetesimals in the Solar Nebula, Icarus, 143, 15-27

[-] Kozlov V.G., Ivanova A.A. and Evesque P., (2006), Block stratification of sedimenting granular matter in a vessel due to vertical vibration, Fluid Dyn. Mater. Process., 2(3), 203-210.

[-] Kozman, I. and J. Gavis, (1962), Development of Charge in Low Conductivity Liquids Flowing Past Surfaces: Experimental Verification and Application of the Theory Developed for Tube Flow, Chem. Eng. Sci., 1062(17), 10231040 .

[-] Kuhlmann H. C. and Rath H. J., (1993), On the interpretation of phase measurements of oscillatory thermocapillary convection in liquid bridges, Phys. Fluids, 5 (9), 2117-2120.

[-] Kuhlmann H.C., Lappa M., Melnikov D., Mukin R., Muldoon F.H., Pushkin D., Shevtsova V.S., and Ueno I., (2014), The JEREMI-Project on thermocapillary convection in liquid bridges. Part A: Overview of Particle Accumulation Structures, Fluid Dyn. Mater. Process., 10 (1), 1-36.

[-] Lamb H. (1932), Hydrodynamics, Dover, New York.

[-] Lan, C.W., Liang, M.C. and Chian, C.H., (2000), Suppressing three-dimensional unsteady flows in vertical zonemelting by steady ampoule rotation, J. Cryst. Growth, 213 (3-4), 395-407.

[-] Langbein D., (1991), Motion of Ensembles of Spherical Particles in a Fluid Due to g-jitter, Adv. Space Res., 11(7), $189-196$.

[-] Lappa M., (2003), Three-dimensional numerical simulation of Marangoni flow instabilities in floating zones laterally heated by an equatorial ring, Phys. Fluids, 15 (3), 776-789.

[-] Lappa M., (2004), Fluids, Materials and Microgravity: Numerical Techniques and Insights into the Physics, Elsevier Science (2004, Oxford, England)

[-] Lappa M. and Carotenuto L., (2003), Effect of convective disturbances induced by g-jitter on the periodic precipitation of lysozyme, Microgravity Sci. Tech., XIV/2, 41-56.

[-] Lappa M., Piccolo C., Carotenuto L., (2004), Mixed buoyant-Marangoni convection due to dissolution of a droplet in a liquid-liquid system with miscibility gap, European Journal of Mechanics/B Fluids, 23/5, 781-794

[-] Lappa M., (2005) Assessment of VOF Strategies for the analysis of Marangoni Migration, Collisional Coagulation of Droplets and Thermal wake effects in Metal Alloys under Microgravity conditions, Computers, Materials \& Continua CMC, 2(1), 51-64. 
[-] Lappa M., (2011), A theoretical and numerical multiscale framework for the analysis of pattern formation in protein crystal engineering, Int. J. Multiscale Comp. Eng., 9 (2), 149-174.

[-] Lappa M., (2013a), On the variety of particle accumulation structures under the effect of gjitters, J. Fluid Mech., 726, 160-195.

[-] Lappa M., (2013b), Assessment of the role of axial vorticity in the formation of Particle Accumulation Structures in supercritical Marangoni and hybrid thermocapillary-rotation-driven flows, Phys. Fluids, 25(1), 012101.

[-] Lappa M., (2013c), On the Existence and Multiplicity of One-dimensional Solid Particle Attractors in Timedependent Rayleigh-Bénard Convection, Chaos, 23(1), 013105. DOI: 10.1063/1.4773001

[-] Lappa M., (2014a), Stationary Solid Particle Attractors in Standing Waves, Physics of Fluids, $26(1), 013305$ (12 pages).

[-] Lappa M., (2014b), The patterning behaviour and accumulation of spherical particles in a vibrated non-isothermal liquid, Physics of Fluids, 26(9), 093301 (22 pages).

[-] Lappa M., (2015), Control of convection patterning and intensity in shallow cavities by harmonic vibrations, Microgravity Science \& Technology, 28(1), 29-39.

[-] Lappa M., Rotating Thermal Flows in Natural and Industrial Processes (John Wiley \& Sons, Chichester, England, 2012).

[-] Lappa M., Savino R. and Monti R., (2001), Three-dimensional numerical simulation of Marangoni instabilities in liquid bridges: influence of geometrical aspect ratio, Int. J. Num. Meth. Fluids, 36 (1): 53-90

[-] Lappa M., Thermal Convection: Patterns, Evolution and Stability (John Wiley \& Sons, Chichester, England, 2009).

[-] Lappa M., (2016), A Mathematical and Numerical Framework for the Analysis of Compressible Thermal Convection in Gases at very high Temperatures, Journal of Computational Physics, 313, 687-712.

[-] Lee H.M. and Park O.O., (1994), Rheology and dynamics of immiscible polymer blends, Journal of Rheology, 38, 1405-1425.

[-] Lin D. N. C., and Papaloizou J., (1980), On the structure and evolution of the primordial solar nebula, Mon. Not. R. Astron. Soc., 191, 37-48.

[-] Longuet-Higgins M. and Gill A., (1967), Resonant interactions between planetary waves, Proc. Roy. Soc. A 229, 120-144.

[-] Love S.G. and Pettit D.R., (2004), Fast repeteable clumping of solid particles in microgravity, Lunar and Planetary Science, XXXV (2004)

[-] Lovelace R. V. E., Li H., Colgate S. A. and Nelson A. F., (1999), Rossby wave instability of Keplerian accretion disks, ApJ., 513, 805-810

[-] Lynden-Bell D. and Pringle J. E., (1974), The evolution of viscous discs and the origin of the nebular variables, Mon. Not. R. Astron. Soc. 168, 603-637.

[-] Manley S., Cipelletti L., Trappe V., Bailey A. E., Christianson R. J., Gasser U., Prasad V., Segre P. N., Doherty M. P., Sankaran S., Jankovsky A. L., Shiley B., Bowen J., Eggers J., Kurta C., Lorik T., and Weitz D. A., (2004), Limits to Gelation in Colloidal Aggregation, Phys. Rev. Lett. 93, 108302.

[-] Mason G., (1999), New fundamental concepts in emulsion rheology, Curr. Opin. Colloid Interface Sci., 4, $231-238$.

[-] Maxey M. R. and Riley J. J., (1983), Equation of motion for a small rigid sphere in a nonuniform flow, Phys. Fluids, 26, 883-889.

[-] Maxey M.R., Patel B.K., Chang E.J., Wang L.-P., (1997), Simulations of dispersed turbulent multiphase flow, Fluid Dyn. Res., 20(1-6), 143-156.

[-] Meneguz E. and Reeks M. W., (2011), Statistical properties of particle segregation in homogeneous isotropic turbulence, J. Fluid Mech., 686: 338-351.

[-] Moisy F., Doaré O., Pasutto T., Daube O., and Rabaus M., (2004), Experimental and numerical study of the shear layer instability between two counter-rotating disks, J. Fluid Mech., 507, 175-202.

[-] Monti R., Savino R., Lappa M., (1998), Microgravity sensitivity of typical fluid physics experiment, presented at the $17^{\text {th }}$ Microgravity Measurements Group Meeting, Cleveland, Ohio, 24-26 March 1998, published in the meeting proceedings in NASA CP-1998-208414, 23, pp. 1-15 (ISSN: 0191-7811).

[-] Monti R., Savino R., Lappa M., (2001), On the convective disturbances induced by g-jitter on the space station, Acta Astronautica, 48 (5-12), 603-615.

[-] Ozaki M., Egami T., Sugiyama N., Matijevića E., (1988), Agglomeration in colloidal hematite dispersions due to weak magnetic interactions: II. The effects of particle size and shape, Journal of Colloid and Interface Science, 126(1), 212-219.

[-] Pacheco-Martinez H. A., Liao L., Hill R. J. A., Swift M.R., and Bowley R. M., (2013), Spontaneous Orbiting of Two Spheres Levitated in a Vibrated Liquid, Phys. Rev. Lett. 110, 154501.

[-] Parmar M., Haselbacher A. and Balachandar S., (2012), Equation of motion for a sphere in non-uniform compressible flows, J. Fluid Mech., 699, 352-375.

[-] Petit L. and Noetinger B., (1988), Shear induced structures in macroscopic dispersions, Rheol. Acta, 27(4), 437-441.

[-] Preisser F., Schwabe D. and Scharmann A., (1983) Steady and oscillatory thermocapillary convection in liquid columns with free cylindrical surface, J. Fluid Mech., 126, 545-567 
[-] Prinz B., Romero A., (1993), New Casting Process for Hypermonotectic Alloys , in Immiscible liquid metals and organics, Proceedings of an international workshop organized by the ESA expert working group, "Immiscible alloys" held in the Physikzentrum, Bad Honnef 1992, edited by L. Ratke, 1993, Germany, 281-289.

[-] Pushkin D., Melnikov D., Shevtsova V., (2011), Ordering of Small Particles in One-Dimensional Coherent Structures by Time-Periodic Flows, Phys. Rev. Lett., 106, 234501.

[-] Raju N. and Meiburg E., (1995), The accumulation and dispersion of heavy particles in forced two-dimensional mixing layers. Part 2: The effect of gravity, Phys. Fluids, 7, 1241-1264.

[-] Ratke L., (1992), "Decomposition, Phase separation, Solidification of immiscible liquid alloys", in Immiscible liquid metals and organics, Proceedings of an international workshop organized by the ESA expert working group, "Immiscible alloys" held in the Physikzentrum, Bad Honnef 1992, edited by L. Ratke, (Informationsgesellschaft, Verlag, Germany, 1992), 3-34.

[-] Ratke L., Korekt G., Drees S., (1996), Solidification of immiscible alloys, ESA SP 385, pag. 247.

[-] Ravichandran S., Perlekar P. and Govindarajan R., (2014), Attracting fixed points for heavy particles in the vicinity of a vortex pair, Phys. Fluids 26, 013303.

[-] Rhines P. B., (1975), Waves and turbulence on a beta-plane,” J. Fluid Mech. 69, 417-443.

[-] Rossby, C-G., (1940), Planetary flow patterns in the atmosphere, Quart. J. Roy. Meteor. Soc. (suppl), 66, 68-87.

[-] Roths T., Friedrich C., Marth M., Honerkamp J., (2002), Dynamics and rheology of the morphology of immiscible polymer blends - on modeling and simulation, Journal Rheologica Acta , 41(3), 211-222.

[-] Rozyczka M., Bodenheimer P., and Bell K. R., (1994), A numerical study of viscous flows in axisymmetric aaccretion disks, Astrophys. J. 423, 736-747.

[-] Ruden S. P., Papaloizou J. C. B., and Lin D. N. C., (1988), Axisymmetric perturbations of thin gaseous disks. I Unstable convective modes and their consequences for the solar nebula, Astrophysical Journal, Part 1, 329, 739-763

[-] Saadatmand M. and Kawaji M., (2010), Effect of Viscosity on Vibration-Induced Motion of a Spherical Particle Suspended in a Fluid Cell, Microgravity Sci. Technol., 22, 433-440,.

[-] Saeedi E., Abbasi S., Bohringer K.F. and Parviz B.A., (2006), Molten-Alloy Driven Self-Assembly for Nano and Micro Scale System Integration, Fluid Dynamics and Materials Processing, 2(4), 221-246.

[-] Sapsis T. and Haller G., (2009), Inertial Particle Dynamics in a Hurricane, J. Atmos. Sci., 66, 2481-2492.

[-] Savino R., Lappa M., (2003), Assessment of the thermovibrational theory: application to g-jitter on the Space-station, Journal of Spacecraft and Rockets, 40 (2), 201-210.

[-] Saw E. W., Shaw R. A., Ayyalasomayajula S., Chuang P. Y., and Gylfason A., (2008), Inertial Clustering of Particles in High-Reynolds-Number Turbulence, Phys. Rev. Lett., 100, 214501.

[-] Schouveiler L., Le Gal P., Chauve M. P., and Takeda Y., (1999), Spiral and circular waves in the flow between a rotating and a stationary disk, Exp. Fluids, 26(3), 179-187.

[-] Schwabe D. and Frank S., (1999), Particle accumulation structures (PAS) in the toroidal thermocapillary vortex of a floating zone - Model for a step in planet-formation?, Advances in Space Research, 23(7), 1191-1196.

[-] Schwabe D., Moller U., Schneider J. and Scharmann A., (1992), Instabilities of shallow dynamic thermocapillary liquid layers, Phys. Fluids A, 4, 2368-2381.

[-] Schwabe D., Hintz P., Frank S., (1996), New Features of Thermocapillary Convection in Floating Zones Revealed by Tracer Particle Accumulation Structures, Microgravity Sci. Tech., 9, 163-168.

[-] Schwabe D., Mizev A.I., Udhayasankar M. and Tanaka S., (2007), Formation of dynamic particle accumulation structures in oscillatory thermocapillary flow in liquid bridges, Phys. Fluids, 19(7), 072102.

[-] Schwabe D. and Mizev A. I., (2011), Particles of different density in thermocapillary liquid bridges under the action of travelling and standing hydrothermal waves, Eur. Phys. J. Spec. Top. 192, 13-27.

[-] Sheehan D. P., Davis S. S., Cuzzi J. N. and Estberg G. N., (1999), Rossby Wave Propagation and Generation in the Protoplanetary Nebula, Icarus, 142(1), 238-248

[-] Shevtsova V., Mialdun A., Kawamura H., Ueno I., Nishino K. and Lappa M., (2011), Onset of Hydrothermal Instability in Liquid Bridge. Experimental Benchmark, Fluid Dynamics \& Materials Processing, 7(1), 1-28

[-] Shi W.Y. and Imaishi N., (2006), Hydrothermal waves in differentially heated shallow annular pools of silicone oil, J. Cryst. Growth, 290, 280-291.

[-] Sun J., Carlson F.M., Regel L.L., Wilcox W.R., Lal R.B., Trolinger J.D., (1994), Particle motion in the Fluid Experiment System in Microgravity, Acta Astronautica, 34, 261-269

[-] Sundaram S. and Collins L.R., (1997), Collision statistics in an isotropic particle laden turbulent suspension. Part 1: Direct numerical simulations, J. Fluid Mech., 335, 75-109.

[-] Tabakova S.S. and Zapruanov Z.D., (1982), On the hydrodynamic interaction of two spheres oscillating in a viscous fluid. I. Axisymmetrical case. J. Appl. Math. Phys. (ZAMP), 33, 344-357 - II. Three dimensional case. J. Appl. Math. Phys. (ZAMP), 33,487-502.

[-] Tanga P., Babiano A., Dubrulle B., and Provenzale A., (1996), Forming Planetesimals in Vortices, Icarus 121, 158170 .

[-] Thomas C.C. and Gollub J.P., (2004), Structures and chaotic fluctuations of granular clusters in a vibrated fluid layer, Phys. Rev. E 70, 061305 
[-] Thomson J.R., Casademunt J., Drolet F. and Vinals J., (1997), Coarsening of solid-liquid mixtures in a random acceleration field, Phys Fluids, 9(5), 1336-1343.

[-] Tirumkudulu M., Tripathi A., Acrivos A., (1999), Particle segregation in monodisperse sheared suspensions, Phys. Fluids, 11(3), 507-509,

[-] Tytell D., (2004), Building Planets in Plastic Bags. Sky and Telescope (SkyandTelescope.com), April 13, 2004

[-] Utracki L.A., (1983), Melt flow of polymer blends, Polymer Engineering and Science, 23(11), $602-609$.

[-] Valverde J. M. and Castellanos A., (2006), Random loose packing of cohesive granular materials, EPL (Europhysics Letters), 75, 6, 985-991.

[-] Voth G. A., Bigger B., Buckley M.R., Losert W., Brenner M.P., Stone H.A., and Gollub J.P., (2002), Ordered clusters and dynamical states of particles in a vibrated fluid, Phys. Rev. Lett., 88, 234301.

[-] Wang L-P., Ayala O., Rosa B., Grabowski W.W., (2008), Turbulent collision efficiency of heavy particles relevant to cloud droplets, New J. Phys., 10: 075013.

[-] Wilkinson M. and Mehlig B., (2005), Caustics in turbulent aerosols, Europhys.Lett., 71, 186-192.

[-] Wilkinson M., Mehlig B., Ostlund S.and Duncan K. P., (2007), Unmixing in random flows, Phys. Fluids, 19, 113303.

[-] Williams, G.P., (2003), Jovian dynamics. Part III. Multiple, migrating, and equatorial Jets, J. Atmos. Sci. 60, 12701296.

[-] Wunenburger R., Carrier V., and Garrabos Y., (2002), Periodic order induced by horizontal vibrations in a twodimensional assembly of heavy beads in water, Phys. Fluids, 14(7), 2350-2359.

[-] Yuan H. G., Kalfas G. \& Ray W. H., (1991), Suspension Polymerization, Journal of Macromolecular Science, Part

C: Polymer Reviews, 31(2-3), 215-299

[-] Zhu Y-J. and Ma Y-Q., (2002), Orientational structures of a phase-separating system under oscillatory particles, J. Chem. Phys. 117, 10207.

[-] Zhu C., Yu Q., Dave R.N., Pfeffer R., (2005), Gas fluidization characteristics of nanoparticle agglomerates, AIChE Journal, 51(2), 426-439. 